\title{
Table and Mollier Chart of the Thermodynamic Properties of 1, 3-Butadiene*
}

\author{
By Cyril H. Meyers, Carl S. Cragoe, and Eugene F. Mueller
}

\begin{abstract}
This paper contains a working table and Mollier chart of the thermodynamic properties of 1,3-butadiene in engineering units, for which the data were calculated from a set of empirical equations. These equations have been published elsewhere and shown to be consistent with exact thermodynamic relations and to represent the observed data within the limits of the experimental accuracy. The table covers the properties of the saturated fluid over the temperature range extending from the triple point $\left(-164.05{ }^{\circ} \mathrm{F}\right)$ to within 20 degrees of the critical temperature $\left(305.6^{\circ} \mathrm{F}\right)$. Examples are solved to illustrate the use of the table and Mollier chart $(\log P$ versus $H)$. Several other charts are also presented.
\end{abstract}

\section{Introduction}

The extensive use of butadiene in the production of synthetic rubber led to a need for more complete and more accurate data on the properties of this substance than were available when it acquired commercial and military importance. Letter Circular LC710, a collection of the available data at the time, was published in December 1942. Further need developed for tables of liquid densities of butadiene and other $\mathrm{C}_{4}$ hydrocarbons found in commercial $\mathrm{C}_{4}$ mixtures, for the purpose of computing, from observed volumes, the quantities of materials bought or sold. This problem was brought to the National Bureau of Standards in July 1943, by representatives of the Rubber Reserve Company. Letter Circular LC 736 , containing tables of liquid densities of eleven hydrocarbons found in commercial $\mathrm{C}_{4}$ mixtures, was prepared from data available in the literature and issued in November 1943. The need for more extensive tables of the thermodynamic properties of butadiene led to a program of experimental work at the National Bureau of Standards to supply the data. The results of these measurements were published in 1945 in Research Papers RP1640 and RP1661. The present paper gives the data in the customary engineering units in the more useful form of an extensive table and a Mollier chart.

\footnotetext{
*This paper was also published as a Technical Report to the Office of Rubber Reserve, June 30, 1947.
}

\section{Experimental Data}

The experimental data upon which these tables are based are those given in two recent publications $[7,8]^{1}$. These publications describe the results of measurements made on butadiene at the National Bureau of Standards, compare them with the relatively small amount of data available from other laboratories, and develop empirical equations that are consistent with exact thermodynamic relations and that represent the observed data within the limit of experimental accuracy, which for P-V-T data on the vapor is one to three parts per thousand, for density of the liquid a very few parts in ten thousand, and for the excess of the enthalpy or entropy over that for the solid at the triple point is approximately one part per thousand. This limit is probably as small as is practicable in view of the rather unstable character of the material that polymerizes to some extent, even while observations are in progress.

Other publications $[1,2]$ were consulted, and the data they contained were used for comparison and verification, but not to any considerable extent as source data for the tables. The temperature of the triple point was taken as $-164.05^{\circ} \mathrm{F}$ $\left(-108.92^{\circ} \mathrm{C}\right)$, that of the boiling point as $24.06^{\circ} \mathrm{F}$ $\left(-4.41^{\circ} \mathrm{C}\right)$, and that of the critical point as $305.6^{\circ} \mathrm{F}\left(152^{\circ} \mathrm{C}\right)$. The value for the critical point is believed to be reliable to one or two ${ }^{\circ} \mathrm{C}$. Poly-

${ }^{1}$ Figures in brackets indicate the literature references at the end of this paper. 
merization occurring before and during the observations limited the accuracy of the determination of the critical temperature.

\section{Notation}

In general, the abbreviations approved as American Tentative Standard (ASA Z101-1932) have been used, with the exception that in giving numerica: values of temperatures, the degree $\operatorname{sign}\left({ }^{\circ}\right)$ has been retained. The units and symbols used are listed in table 1.

\begin{tabular}{l} 
TABLE 1._Units and notation \\
\hline \multicolumn{1}{c|}{ Quantity } \\
\hline Pressure
\end{tabular}

SUBSCRIPTS
Property of liquid.
Property of vapor
Transition from liquid to vapor

$a$ The factor $1\left(\mathrm{ft}^{3}\right)\left(\mathrm{lb} / \mathrm{in}^{2}\right)=0.185052 \mathrm{Btu}$ is necessary for numerical calculations in engineering units.

\section{Fundamental Units and Constants \\ 1. Temperature Scale}

The Fahrenheit scale used in these tables is derived from the international temperature scale [3] of 1927 by means of the relation:

$$
\begin{gathered}
\text { Fahrenheit temperature }=1.8 \times \text { centigrade } \\
\text { temperature }+32 .
\end{gathered}
$$

It is not expected that any changes that may be made when the pending revision of the scale of 1927 is adopted, will require any material change in the numerical values of temperatures as given in these tables.

Temperatures on the absolute Fahrenheit scale were obtained by adding $459.688^{\circ}$ to the temperatures as defined above.

\section{Heat Units}

All of the calorimetric measurements were made in terms of the international joule, which was therefore the fundamental heat unit. It is based upon the standards of resistance and electromotive force maintained at the Bureau and upon the mean solar second. The relation between the international joule and the corresponding cgs units, according to the most recent data [4] is:

1 international joule $=1.00017$ absolute joules.

In the thermodynamic equations relations occur between quantities of energy, some of which were measured in international joules, whereas others such as the product of pressure and volume each expressed in appropriate units, were measured directly in mechanical units, that is, absolute joules. Although the difference might be considered of negligible importance, it was taken in to account where it could have any appreciable effect on the results obtained.

As secondary heat units the calorie and the British thermal unit (Btu) have been used. The calorie is the International Table (IT) calorie, widely used in engineering tables of the properties of steam. It was officially adopted by the second International Steam Table conference [5] held in London in 1929, and is defined as $3,600 / 860$ (approximately 4.1860) international joules. In this way the mechanical equivalent of heat, so significant in earlier tables, loses its importance as it becomes a defined rather than an experimental constant and therefore is in no way dependent upon the results of past or future measurements of the properties of water.

The Btu used is derivable from the calorie by use of relations that depend only upon the relative sizes of the centigrade and Fahrenheit degrees, and the relation between the kilogram and the pound thus,

$$
1 \quad \text { Btu }=453.592 \times \frac{5}{9}=251.996 \quad \text { IT } \quad \text { calories }=
$$
1054.866 int joules. This method of defining the $\mathrm{B}$ tu retains the convenient relation

1 calorie per gram=1.8 Btu per pound. 


\section{Relations Between Energy Units}

The relation between the Btu defined above, and the standard foot-pound is $1 \mathrm{Btu}=1054.866$ int joules $\times \frac{10^{7} \times 0.3937}{980.665 \times 453.592 \times 12}=778.160 \mathrm{ft} \mathrm{lb}$. In using the tables, quantities of heat or work that are given in Btu may be converted into foot-pounds by multiplying by the factor 778.160. In cases where the reverse conversion is desired, the relation mav be written,

$$
1 \mathrm{ft}-\mathrm{lb}=0.00128508 \text { Btu. }
$$

As volumes are given in $\mathrm{ft}^{3}$ and pressures in $\mathrm{lb} / \mathrm{in}^{2}$, the relation $1\left(\mathrm{ft}^{3}\right)\left(\mathrm{lb} / \mathrm{in}^{2}\right)=144(0.00128508)=$ $0.185052 \mathrm{Btu}$ becomes necessary wherever numerical values are given in an equation involving both energy in Btu and the product $p v$ in British engineering units.

\section{Units of Pressure}

In the British system the unit of force is the pound weight, which becomes definite when the value of the acceleration due to gravity is specified. The value used here is $980.665 \mathrm{~cm} / \mathrm{sec}^{-2} .^{2}$

The unit of pressure used in this publication is one standard pound weight per sq in. absolute. The lower pressures have been expressed also in inches of mercury with use of the factor,

$$
1 \mathrm{lb} / \mathrm{in}^{2}=2.03601 \text { in } \mathrm{Hg} .
$$

\footnotetext{
2 In 1901 the International Committee of Weights and Measures recom. mended the adoption of an experimental value $980.665 \mathrm{~cm} / \mathrm{sec}^{2}\left(32.174 \mathrm{ft} / \mathrm{sec}^{2}\right)$ as the value of gravity at latitude $45^{\circ}$ and sea level [5]. Since then, those who have occasion to use a standard value for $g$ have been divided into two groups, one of which uses the current "best" value for $g$ at latitude $45^{\circ}$ and sea level. The current value is about $980.62 \mathrm{~cm} / \mathrm{sec}^{2}$. The other group has loyally fol. lowed the lead of the international eommittee in using $980.665 \mathrm{~cm} / \mathrm{sec}^{2}$. The value $980.665 \mathrm{~cm} / \mathrm{sec}^{2}$ has been used in computing these tables.
}

\section{Units of Volume and Density}

The volumetric apparatus was calibrated either with water or with mercury. The volume unit was defined by the relations:

One $\mathrm{cm}^{3}$ of water at $4^{\circ} \mathrm{C}$ under 1 atm pressure has a mass of $0.999973 \mathrm{~g}$. One $\mathrm{ml}$ of water at $4^{\circ} \mathrm{C}$ under $1 \mathrm{~atm}$ pressure has a mass of 1.000000 grams. One $\mathrm{cm}^{3}$ of mercury at $0^{\circ} \mathrm{C}$ under 1 atm pressure has a mass of $13.5951 \mathrm{~g}$. Densities measured in $\mathrm{g} / \mathrm{ml}$ were converted by means of the relation [4],

$1 \mathrm{~g} / \mathrm{ml}=62.42658 \mathrm{lb} / \mathrm{ft}^{3}$.

\section{Description of Table}

Only the properties of the saturated fluid have been tabulated. These are presented in table 2, which uses the notation described in the preceding section and in which the argument is temperature. The table covers the whole temperature range from the triple point $-164.05^{\circ} \mathrm{F}$ to the critical temperature $305.6^{\circ} \mathrm{F}$. Values are given every degree Fahrenheit in the temperature range $0^{\circ}$ to $200^{\circ} \mathrm{F}$ and at less frequent intervals outside this range. Such arrangement gives a table with an ample number of entries in the portion most used, and keeps the table within a reasonable size. The pressures are absolute and for the lower temperatures are given in two units, namely $\mathrm{lb} / \mathrm{in}^{2}$ and inches $\mathrm{Hg}$ at $32^{\circ} \mathrm{F}$. The next two columns are devoted to the specific volume of liquid and vapor respectively in $\mathrm{ft}^{3} / \mathrm{lb}$. In that portion of the table where only one column of pressures is given, a column of densities of the vapor in $\mathrm{lb} / \mathrm{ft}^{3}$ has been inserted so that the table contains the same number of columns throughout. 
TABLE 2.-Properties of saturated 1,3-butadiene

\begin{tabular}{|c|c|c|c|c|c|c|c|c|c|c|}
\hline \multirow{2}{*}{$\begin{array}{l}\text { Tempera- } \\
\text { ture, } t\end{array}$} & \multicolumn{2}{|c|}{ Abs. pressure } & \multicolumn{2}{|c|}{ Specific volume } & \multicolumn{3}{|c|}{ Enthalpy } & \multicolumn{2}{|c|}{ Entropy } & \multirow{2}{*}{$\begin{array}{l}\text { Tempera- } \\
\text { ture, } t\end{array}$} \\
\hline & $p$ & $p$ & Liquid, $v_{f}$ & Vapor, $v_{g}$ & Liquid, $h_{f}$ & Evap., $h_{f g}$ & Vapor, $h_{g}$ & Liquid, $s_{f}$ & Vapor, $s_{g}$ & \\
\hline${ }^{\circ} F$ & $l b / i n .^{2}$ & in. $\mathrm{Hg}$ & $f t^{3} / l b$ & $f t^{3} / l b$ & $B t u / l b$ & $B t u / l b$ & $B t u / l b$ & Ptu/lb $b^{\circ} R$ & $P t u / l b^{\circ} R$ & ${ }^{\circ} F$ \\
\hline Triple & 0.010 & Solid & 8. & 5,706 & 59. 14 & 282.7 & 341.8 & 0.3757 & 1. 3317 & -164.05 \\
\hline point & .010 & Liquid & 0.020972 & 5,706 & 122.61 & 219.2 & 341.8 & .5904 & 1. 3317 & -164.05 \\
\hline-160 & .013 & 0.027 & .021036 & 4,504 & 124.44 & 218.3 & 342.7 & .5973 & 1. 3256 & -160 \\
\hline-155 & .018 & .037 & .021117 & 3,310 & 126. 71 & 217.1 & 343.8 & .6048 & 1. 3174 & -155 \\
\hline-150 & .025 & .051 & .021198 & 2,461 & 128.98 & 216.0 & 345.0 & .6122 & 1. 3096 & -150 \\
\hline-145 & .034 & .069 & .021279 & 1,850 & 131. 26 & 214.8 & 346.1 & .6195 & 1. 3023 & -145 \\
\hline-140 & .045 & .092 & .021362 & 1,406 & 133.55 & 213.7 & 347.3 & .6267 & 1. 2953 & -140 \\
\hline-135 & .060 & .121 & .021446 & $1,079.6$ & 135.83 & 212.6 & 348.4 & .6338 & 1. 2886 & -135 \\
\hline-130 & .078 & .159 & .021530 & 836.8 & 138.13 & 211.5 & 349. 6 & .6408 & 1. 2823 & -130 \\
\hline-125 & .101 & .206 & .021615 & 654.6 & 140.42 & 210.4 & 350.8 & .6478 & 1. 2764 & -125 \\
\hline-120 & .130 & .265 & .021701 & 516.5 & 142.72 & 209.3 & 352.0 & .6546 & 1. 2707 & -120 \\
\hline-115 & .166 & .338 & .021787 & 411.0 & 145.03 & 208.2 & 353.2 & .6613 & 1. 2654 & -115 \\
\hline-110 & .210 & .428 & .021876 & 329.6 & 147.33 & 207.1 & 354.5 & .6680 & 1. 2603 & -110 \\
\hline-105 & .264 & .537 & .021964 & 266.3 & 149.65 & 206.1 & 355.7 & .6745 & 1. 2555 & -105 \\
\hline-100 & .329 & .669 & .022054 & 216.7 & 151.96 & 205.0 & 356. 9 & .6810 & 1. 2509 & -100 \\
\hline-95 & .407 & .828 & .022144 & 177.6 & 154. 29 & 203.9 & 358. 2 & .6874 & 1. 2466 & -95 \\
\hline-90 & .500 & 1. 017 & .022236 & 146.4 & 156.61 & 202.9 & 359.5 & .6938 & 1. 2425 & -90 \\
\hline-85 & .610 & 1. 242 & .022328 & 121. 52 & 158.95 & 201.8 & 360.8 & .7000 & 1. 2386 & -85 \\
\hline-80 & .740 & 1. 507 & .022420 & 101.44 & 161. 29 & 200.8 & 362.0 & .7062 & 1. 2350 & -80 \\
\hline-75 & .893 & 1.817 & .022511 & 85.16 & 163.63 & 199. 7 & 363. 3 & .7124 & 1. 2315 & -75 \\
\hline-70 & 1. 071 & 2. 180 & .022606 & 71.88 & 165.99 & 198. 7 & 364.7 & .7184 & 1. 2283 & -70 \\
\hline-65 & 1. 277 & 2. 600 & .022702 & 60.98 & 168.35 & 197. 6 & 366.0 & .7245 & 1. 2252 & -65 \\
\hline-60 & 1.516 & 3. 086 & .022799 & 52.00 & 170.72 & 196.6 & 367.3 & .7304 & 1. 2223 & -60 \\
\hline-58 & 1. 621 & 3. 300 & .022838 & 48.85 & 171.67 & 196. 2 & 367.8 & .7328 & 1. 2212 & -58 \\
\hline-56 & 1. 732 & 3. 526 & .022878 & 45.93 & 172.62 & 195.8 & 368.4 & .7352 & 1. 2201 & -56 \\
\hline-54 & 1. 849 & 3. 765 & .022917 & 43. 21 & 173.57 & 195.3 & 368.9 & .7375 & 1. 2190 & -54 \\
\hline-52 & 1.973 & 4. 017 & .022957 & 40.68 & 174.53 & 194.9 & 369.5 & .7399 & 1. 2180 & -52 \\
\hline-50 & 2. 103 & 4. 283 & .022997 & 38.33 & 175.49 & 194.5 & 370.0 & .7422 & 1. 2170 & -50 \\
\hline-48 & 2. 241 & 4. 563 & .023037 & 36.14 & 176.44 & 194.1 & 370.5 & .7445 & 1. 2160 & -48 \\
\hline-46 & 2. 386 & 4.857 & .023077 & 34.10 & 177.40 & 193.7 & 371.1 & .7469 & 1. 2150 & -46 \\
\hline-44 & 2. 538 & 5. 167 & .023118 & 32.19 & 178. 36 & 193.3 & 371.6 & .7492 & 1. 2141 & -44 \\
\hline-42 & 2. 698 & 5. 494 & .023158 & 30.41 & 179.33 & 192.8 & 372.2 & .7515 & 1. 2132 & -42 \\
\hline-40 & 2. 867 & 5. 836 & .023199 & 28.75 & 180. 29 & 192.4 & 372.7 & .7538 & 1. 2123 & -40 \\
\hline-38 & 3. 043 & 6. 196 & .023240 & 27.20 & 181. 26 & 192.0 & 373.3 & .7561 & 1. 2114 & -38 \\
\hline-36 & 3. 229 & 6. 574 & .023281 & 25.74 & 182.23 & 191.6 & 373.8 & .7584 & 1. 2105 & -36 \\
\hline-34 & 3.423 & 6. 970 & .023323 & 24.38 & 183.19 & 191. 2 & 374.4 & .7606 & 1. 2097 & -34 \\
\hline-32 & 3.627 & 7. 386 & .023364 & 23.10 & 184.16 & 190.7 & 374.9 & .7629 & 1. 2089 & -32 \\
\hline-30 & 3.841 & 7.821 & .023406 & 21.91 & 185.14 & 190.3 & 375.5 & .7652 & 1. 2081 & -30 \\
\hline-28 & 4. 065 & 8. 277 & .023448 & 20.79 & 186. 11 & 189.9 & 376.0 & .7674 & 1. 2074 & -28 \\
\hline-26 & 4. 299 & 8. 754 & 023491 & 19. 73 & 187.09 & 189.5 & 376.6 & .7697 & 1. 2066 & -26 \\
\hline-24 & 4. 544 & 9. 252 & .023533 & 18.74 & 188.06 & 189.1 & 377. 1 & .7719 & 1. 2059 & -24 \\
\hline-22 & 4.801 & 9.774 & .023576 & 17.81 & 189.04 & 188.6 & 377.7 & .7742 & 1. 2052 & -22 \\
\hline-20 & 5. 068 & 10. 319 & .023619 & 16.94 & 190.02 & 188. 2 & 378. 2 & .7764 & 1. 2045 & -20 \\
\hline-18 & 5. 348 & 10.888 & .023662 & 16.11 & 191.01 & 187.8 & 378.8 & .7786 & 1. 2038 & -18 \\
\hline-16 & 5. 639 & 11. 482 & .023705 & 15. 34 & 191.99 & 187.4 & 379.4 & .7809 & 1. 2032 & -16 \\
\hline-14 & 5. 944 & 12.102 & .023749 & 14.61 & 192.98 & 186.9 & 379.9 & .7831 & 1. 2025 & -14 \\
\hline-12 & 6.261 & 12.748 & .023793 & 13.92 & 193.97 & 186.5 & 380.5 & .7853 & 1. 2019 & -12 \\
\hline-10 & 6. 592 & 13. 421 & .023837 & 13. 27 & 194. 96 & 186.1 & 381.0 & .7875 & 1. 2013 & -10 \\
\hline-8 & 6.937 & 14. 123 & .023882 & 12.66 & 195.95 & 185.7 & 381.6 & .7897 & 1. 2007 & -8 \\
\hline-6 & 7.295 & 14.854 & .023926 & 12.08 & 196. 94 & 185. 2 & 382.2 & .7919 & 1. 2002 & -6 \\
\hline-4 & 7. 669 & 15. 614 & .023971 & 11.53 & 197.94 & 184.8 & 382.7 & .7941 & 1. 1996 & -4 \\
\hline-2 & 8.057 & 16.405 & .024016 & 11.01 & 198.94 & 184.4 & 383.3 & .7963 & 1. 1991 & -2 \\
\hline 0 & 8.461 & 17. 23 & .024062 & 10.525 & 199. 94 & 183.9 & 383.9 & .7984 & 1. 1985 & 0 \\
\hline 1 & 8. 669 & 17.65 & .024084 & 10. 290 & 200.44 & 183.7 & 384.2 & .7995 & 1. 1983 & 1 \\
\hline 2 & 8. 881 & 18.08 & .024107 & 10. 062 & 200.94 & 183.5 & 384.4 & .8006 & 1. 1980 & 2 \\
\hline 3 & 9. 097 & 18.52 & .024130 & 9.839 & 201.44 & 183.3 & 384.7 & .8017 & 1. 1978 & 3 \\
\hline 4 & 9. 317 & 18.97 & .024153 & 9. 623 & 201.94 & 183.1 & 385.0 & .8028 & 1. 1976 & 4 \\
\hline
\end{tabular}


TABLE 2.--Properties of saturated 1,3-butadiene-Continued

\begin{tabular}{|c|c|c|c|c|c|c|c|c|c|c|}
\hline \multirow{2}{*}{$\begin{array}{l}\text { Tempera- } \\
\text { ture, } t\end{array}$} & \multicolumn{2}{|c|}{ Abs. pressure } & \multicolumn{2}{|c|}{ Specific volume } & \multicolumn{3}{|c|}{ Enthalpy } & \multicolumn{2}{|c|}{ Entropy } & \multirow{2}{*}{$\begin{array}{l}\text { Tempera- } \\
\text { ture, } t\end{array}$} \\
\hline & $p$ & $p$ & Liquid, $v_{f}$ & Vapor, $v_{g}$ & Liquid, $h_{f}$ & Evap., $h_{f g}$ & Vapor, $h_{g}$ & Liquid, $s_{f}$ & Vapor, $s_{g}$ & \\
\hline${ }^{\circ} \mathrm{F}$ & lb/in..$^{2}$ & in. $\mathrm{Hg}$ & $f t^{3 / l b}$ & $\mathrm{ft}^{3 / \mathrm{lb}}$ & & Btu/lb & Ptu/lb & Btu/lb $b^{\circ} R$ & $R t u / l b^{\circ} R$ & ${ }^{\circ} \mathrm{F}$ \\
\hline 5 & $\begin{array}{l}9.542 \\
9.770\end{array}$ & $\begin{array}{l}19.43 \\
19.89\end{array}$ & $\begin{array}{r}0.024176 \\
.024199\end{array}$ & $\begin{array}{l}9.413 \\
9.208\end{array}$ & $\begin{array}{l}202.44 \\
202.95\end{array}$ & $\begin{array}{l}182.8 \\
182.6\end{array}$ & $\begin{array}{l}385.3 \\
385.6\end{array}$ & $\begin{array}{r}0.8038 \\
8049\end{array}$ & 1. 1973 & $\begin{array}{l}5 \\
6\end{array}$ \\
\hline $\begin{array}{l}6 \\
7\end{array}$ & $\begin{array}{r}9.770 \\
10.003\end{array}$ & $\begin{array}{l}19.89 \\
20.37\end{array}$ & $\begin{array}{l}.024199 \\
.024223\end{array}$ & $\begin{array}{l}9.208 \\
9.008\end{array}$ & $\begin{array}{l}202.95 \\
203.45\end{array}$ & $\begin{array}{l}182.6 \\
182.4\end{array}$ & $\begin{array}{l}385.6 \\
385.9\end{array}$ & $\begin{array}{r}.8049 \\
.8060\end{array}$ & $\begin{array}{l}1.1971 \\
1.1969\end{array}$ & $\begin{array}{l}6 \\
7\end{array}$ \\
\hline $\begin{array}{l}7 \\
8\end{array}$ & 10. 241 & 20.85 & .024246 & 8.814 & 203. 96 & 182.2 & 386.1 & .8071 & $\begin{array}{l}1.1909 \\
1.1966\end{array}$ & 8 \\
\hline 9 & 10.482 & 21.34 & .024269 & 8.625 & 204.47 & 182.0 & 386.4 & .8082 & 1. 1964 & 9 \\
\hline 10 & 10. 728 & 21.84 & .024293 & 8.441 & 204.97 & 181.7 & 386.7 & .8092 & 1. 1962 & 10 \\
\hline 11 & 10. 979 & 22.35 & .024316 & 8. 261 & 205.48 & 181.5 & 387.0 & .8103 & 1. 1960 & 11 \\
\hline 12 & 11. 234 & 22.87 & .024340 & 8.087 & 205. 98 & 181. 3 & 387.3 & .8114 & 1. 1957 & 12 \\
\hline 13 & 11.494 & 23.40 & .024363 & 7.917 & 206. 49 & 181.1 & 387.6 & .8125 & 1. 1955 & 13 \\
\hline 14 & 11. 759 & 23.94 & .024387 & 7.751 & 207.00 & 180.9 & 387.9 & .8135 & 1. 1953 & 14 \\
\hline 15 & 12. 029 & 24. 49 & .024411 & 7. 589 & 207.50 & 180.6 & 388.1 & .8146 & 1. 1951 & 15 \\
\hline 16 & 12.303 & 25.05 & .024435 & 7.431 & 208. 01 & 180.4 & 388.4 & .8157 & 1. 1949 & 16 \\
\hline 17 & 12. 582 & 25.62 & .024459 & 7. 278 & 208.52 & 180.2 & 388.7 & .8167 & 1. 1947 & 17 \\
\hline 18 & 12.866 & 26.20 & .024483 & 7. 128 & 209.03 & 180.0 & 389.0 & .8178 & 1. 1945 & 18 \\
\hline 19 & 13.155 & 26. 78 & .024507 & 6. 982 & 209.54 & 179.7 & 389.3 & .8189 & 1. 1943 & 19 \\
\hline 20 & 13.45 & 27.38 & .024531 & 6.840 & 210.05 & 179.5 & 389.6 & .8199 & 1. 1942 & 20 \\
\hline 21 & 13.75 & 27.99 & .024555 & 6. 701 & 210.57 & 179.3 & 389.9 & .8210 & 1. 1940 & 21 \\
\hline 22 & 14.05 & 28.61 & .024579 & 6. 566 & 211.08 & 179.1 & 390.1 & .8220 & 1. 1938 & 22 \\
\hline 23 & 14.36 & 29.24 & .024604 & 6.434 & 211. 59 & 178.8 & 390.4 & .8231 & 1. 1936 & 23 \\
\hline 24 & 14.68 & 29.88 & .024628 & 6. 305 & 212.10 & 178.6 & 390.7 & .8242 & 1. 1934 & 24 \\
\hline 25 & 15.00 & 30.54 & .024653 & 6.179 & 212.62 & 178.4 & 391.0 & .8252 & 1. 1933 & 25 \\
\hline 26 & 15.32 & 31.20 & .024677 & 6.057 & 213.13 & 178. 2 & 391.3 & .8263 & 1. 1931 & 26 \\
\hline 27 & 15.66 & 31.87 & .024702 & 5. 937 & 213.65 & 177.9 & 391.6 & .8273 & 1. 1929 & 27 \\
\hline 28 & 15.99 & 32.56 & .024727 & 5.820 & 214.16 & 117. 7 & 391.9 & .8284 & 1. 1928 & 28 \\
\hline 29 & 16.34 & 33.26 & .024752 & 5. 706 & 214.68 & 177.5 & 392.2 & .8294 & 1. 1926 & 29 \\
\hline 30 & 16. 68 & 33.97 & .024777 & 5.595 & 215.19 & 177.2 & 392.4 & .8305 & 1. 1925 & 30 \\
\hline 31 & 17.04 & 34.69 & .024802 & 5.487 & 215.71 & 177.0 & 392.7 & .8316 & 1. 1923 & 31 \\
\hline 32 & 17.40 & 35.42 & .024827 & 5. 381 & 216.23 & 176.8 & 393.0 & .8326 & 1. 1921 & 32 \\
\hline 33 & 17. 76 & 36.17 & .024852 & 5. 277 & 216.75 & 176.6 & 393.3 & .8337 & 1. 1920 & 33 \\
\hline 34 & 18.13 & 36.92 & .024877 & 5. 176 & 217. 27 & 176.3 & 393.6 & .8347 & 1. 1919 & 34 \\
\hline 35 & 18.51 & 37.69 & .024903 & 5.077 & 217. 79 & 176.1 & 393.9 & .8357 & 1. 1917 & 35 \\
\hline 36 & 18.90 & 38.47 & .024928 & 4. 981 & 218. 31 & 175.9 & 394.2 & .8368 & 1. 1916 & 36 \\
\hline 37 & 19. 29 & 39.27 & .024954 & 4. 887 & 218.83 & 175.6 & 394.5 & .8378 & 1. 1914 & 37 \\
\hline 38 & 19. 68 & 40.07 & .024979 & 4. 795 & 219.35 & 175.4 & 394.7 & .8389 & 1. 1913 & 38 \\
\hline 39 & 20.08 & 40.89 & .025005 & 4. 705 & 219.87 & 175. 2 & 395.0 & .8399 & 1. 1912 & 39 \\
\hline 40 & 20.49 & 41. 72 & .025031 & 4. 617 & 220.40 & 174.9 & 395.3 & .8410 & 1. 1910 & 40 \\
\hline 41 & 20.91 & 42.57 & .025057 & 4. 531 & 220.92 & 174.7 & 395. 6 & .8420 & 1. 1909 & 41 \\
\hline 42 & 21.33 & 43.43 & .025083 & 4. 447 & 221.44 & 174.4 & 395.9 & .8431 & 1. 1908 & 42 \\
\hline 43 & 21.76 & 44. 30 & .025109 & 4. 365 & 221.97 & 174. 2 & 396. 2 & .8441 & 1. 1907 & 43 \\
\hline 44 & 22. 19 & 45. 19 & .025135 & 4. 285 & 222.50 & 174.0 & 396.5 & .8451 & 1.1905 & 44 \\
\hline 45 & 22.63 & 46.08 & .025161 & 4. 207 & 223.02 & 173.7 & 396.8 & .8462 & 1. 1904 & 45 \\
\hline 46 & 23.08 & 47.00 & .025188 & 4.130 & 223.55 & 173.5 & 397.0 & .8472 & 1. 1903 & 46 \\
\hline 47 & 23.54 & 47. 92 & .025214 & 4. 055 & 224.08 & 173.3 & 397.3 & .8483 & 1. 1902 & 47 \\
\hline 48 & 24.00 & 48.86 & .025241 & 3.982 & 224.61 & 173.0 & 397.6 & .8493 & 1. 1901 & 48 \\
\hline 49 & 24.47 & 49.82 & .025267 & 3. 911 & 225.13 & 172.8 & 397.9 & .8503 & 1.1900 & 49 \\
\hline
\end{tabular}


TABLe 2.-Properties of saturated 1, 3-butadiene-Continued

\begin{tabular}{|c|c|c|c|c|c|c|c|c|c|c|}
\hline \multirow{2}{*}{$\begin{array}{l}\text { Tempera- } \\
\text { ture, } t\end{array}$} & \multirow{2}{*}{$\begin{array}{l}\text { Abs. } \\
\text { pressure }\end{array}$} & \multicolumn{2}{|c|}{ Specific volume } & \multirow{2}{*}{$\begin{array}{c}\text { Density } \\
\text { vapor } 1 / v_{0}\end{array}$} & \multicolumn{3}{|c|}{ Enthalpy } & \multicolumn{2}{|c|}{ Entropy } & \multirow{2}{*}{$\begin{array}{l}\text { Tempera- } \\
\text { ture, } t\end{array}$} \\
\hline & & $v_{f}$ & $v_{0}$ & & Liquid $h_{f}$ & Evap. $h_{f g}$ & Vapor $h_{g}$ & Liquid $s_{f}$ & Vapor $s_{0}$ & \\
\hline${ }^{\circ} F$ & $\mathrm{lb} / \mathrm{in} .^{2}$ & $\begin{array}{l}f t^{3} / l b \\
0.025294\end{array}$ & $f t^{3} / l b$ & $l b / f^{3}$ & Btu/lb & $B t u / l b$ & Btu/lb & $B t u / l b^{\circ} R$ & $B t u / l b^{\circ} R$ & ${ }^{\circ} \mathrm{F}$ \\
\hline 50 & 24. 94 & 0.025294 & 3.840 & 0.2604 & 225.66 & 172.5 & 398.2 & 0.8514 & 1. 1899 & 50 \\
\hline 51 & 25.43 & .025321 & 3. 772 & .2651 & 226.19 & 172. 3 & 398.5 & .8524 & 1. 1898 & 51 \\
\hline 52 & 25.92 & .025348 & 3.705 & .2699 & 226.72 & 172.1 & 398.8 & .8534 & 1. 1897 & 52 \\
\hline 53 & 26.42 & .025375 & 3.639 & .2748 & 227.26 & 171.8 & 399.1 & .8545 & 1. 1896 & 53 \\
\hline 54 & 26.92 & .025402 & 3.575 & .2797 & 227. 79 & 171.6 & 399.4 & .8555 & 1. 1895 & 54 \\
\hline 55 & 27.43 & .025429 & 3.512 & .2847 & 228.32 & 171.3 & 399.6 & .8565 & 1. 1894 & 55 \\
\hline 56 & 27.95 & .025456 & 3.451 & .2898 & 228.85 & 171.1 & 399.9 & .8575 & 1. 1893 & 56 \\
\hline 57 & 28.48 & .025484 & 3.391 & .2949 & 229.39 & 170.8 & 400.2 & .8586 & 1. 1892 & 57 \\
\hline 58 & 29.01 & .025511 & 3.332 & .3001 & 229. 93 & 170.6 & 400.5 & .8596 & 1. 1891 & 58 \\
\hline 59 & 29.56 & .025539 & 3.275 & .3054 & 230.46 & 170.3 & 400.8 & .8607 & 1. 1891 & 59 \\
\hline 60 & 30.11 & .025567 & 3. 218 & .3107 & 231.00 & 170.1 & 401.1 & .8617 & 1.1890 & 60 \\
\hline 61 & 30.66 & .025595 & 3.163 & .3162 & 231.53 & 169.8 & 401.4 & .8627 & 1. 1889 & 61 \\
\hline 62 & 31.23 & .025623 & 3. 109 & .3217 & 232.07 & 169.6 & 401. 7 & .8637 & 1. 1888 & 62 \\
\hline 63 & 31.80 & .025651 & 3.056 & .3272 & 232.61 & 169.3 & 401. 9 & .8648 & 1. 1887 & 63 \\
\hline 64 & 32.39 & .025679 & 3. 004 & .3329 & 233.15 & 169.1 & 402. 2 & .8658 & 1.1887 & 64 \\
\hline 65 & 32.97 & .025707 & 2. 954 & .3386 & 233.69 & 168.8 & 402.5 & .8668 & 1. 1886 & 65 \\
\hline 66 & 33.57 & .025736 & 2. 904 & .3444 & 234.23 & 168.6 & 402.8 & .8678 & 1. 1885 & 66 \\
\hline 67 & 34.18 & .025764 & 2.855 & .3502 & 234.77 & 168.3 & 403.1 & .8689 & 1. 1885 & 67 \\
\hline 68 & 34.79 & .025793 & 2. 808 & .3562 & 235.31 & 168.1 & 403.4 & .8699 & 1. 1884 & 68 \\
\hline 69 & 35.42 & .025821 & 2. 761 & .3622 & 235.85 & 167.8 & 403. 7 & .8709 & 1. 1883 & 69 \\
\hline 70 & 36.05 & .025850 & 2. 715 & .3683 & 236.40 & 167.6 & 404. 0 & .8719 & 1. 1883 & 70 \\
\hline 71 & 36.69 & .025879 & 2. 670 & .3745 & 236.94 & 167.3 & 404. 2 & .8730 & 1. 1882 & 71 \\
\hline 72 & 37.34 & .025908 & 2. 626 & .3808 & 237.49 & 167.0 & 404.5 & .8740 & 1. 1882 & 72 \\
\hline 73 & 37.99 & .025938 & 2. 583 & .3871 & 238.03 & 166.8 & 404.8 & .8750 & 1. 1881 & 73 \\
\hline 74 & 38.66 & .025967 & 2. 541 & .3935 & 238.58 & 166.5 & 405. 1 & .8760 & 1. 1880 & 74 \\
\hline 75 & 39.33 & .025996 & 2.500 & .4001 & 239.13 & 166.3 & 405.4 & .8770 & 1. 1880 & 75 \\
\hline 76 & 40.02 & .026026 & 2. 459 & .4066 & 239.68 & 166.0 & 405. 7 & .8780 & 1. 1879 & 76 \\
\hline 77 & 40.71 & .026056 & 2.419 & .4133 & 240.23 & 165.7 & 406.0 & .8791 & 1. 1879 & 77 \\
\hline 78 & 41.41 & .026085 & 2. 380 & .4201 & 240.77 & 165.5 & 406.3 & .8801 & 1. 1879 & 78 \\
\hline 79 & 42.12 & .026115 & 2. 342 & .4269 & 241.32 & 165.2 & 406.5 & .8811 & 1. 1878 & 79 \\
\hline 80 & 42.84 & .026145 & 2. 305 & .4339 & 241.88 & 165.0 & 406.8 & .8821 & 1. 1878 & 80 \\
\hline 81 & 43.57 & .026176 & 2. 268 & .4409 & 242.43 & 164.7 & 407.1 & .8831 & 1. 1877 & 81 \\
\hline 82 & 44.31 & .026206 & 2. 232 & .4480 & 242. 98 & 164.4 & 407.4 & .8841 & 1.1877 & 82 \\
\hline 83 & 45.06 & .026236 & 2.197 & .4552 & 243.53 & 164.2 & 407.7 & .8852 & 1. 1876 & 83 \\
\hline 84 & 45.82 & .026267 & 2. 162 & .4625 & 244.09 & 163.9 & 408.0 & .8862 & 1. 1876 & 84 \\
\hline 85 & 46. 59 & .026298 & 2. 128 & .4699 & 244.64 & 163.6 & 408.3 & .8872 & 1. 1876 & 85 \\
\hline 86 & 47.36 & .026328 & 2.095 & .4773 & 245. 20 & 163.4 & 408.5 & .8882 & 1. 1875 & 86 \\
\hline 87 & 48.15 & .026359 & 2. 062 & .4849 & 245.75 & 163.1 & 408.8 & .8892 & 1. 1875 & 87 \\
\hline 88 & 48.95 & .026391 & 2.030 & .4926 & 246. 31 & 162.8 & 409.1 & .8902 & 1. 1875 & 88 \\
\hline 89 & 49. 75 & .026422 & 1. 999 & .5003 & 246.87 & 162.5 & 409. 4 & .8912 & 1.1875 & 89 \\
\hline 90 & 50.57 & .026453 & 1. 968 & .5082 & 247. 43 & 162.3 & 409. 7 & .8922 & 1. $1874^{\circ}$ & 90 \\
\hline 91 & 51.40 & .026485 & 1. 938 & .5161 & 247. 99 & 162.0 & 410.0 & .8933 & 1. 1874 & 91 \\
\hline 92 & 52.23 & .026516 & 1. 908 & .5242 & 248.55 & 161.7 & 410.3 & .8943 & 1. 1874 & 92 \\
\hline 93 & 53.08 & .026548 & 1. 879 & .5323 & 249.11 & 161.4 & 410.5 & .8953 & 1. 1874 & 93 \\
\hline 94 & 53.94 & .026580 & 1. 850 & .5405 & 249.67 & 161.2 & 410.8 & .8963 & 1. 1873 & 94 \\
\hline 95 & 54.81 & .026612 & 1. 822 & .5489 & 250.23 & 160.9 & 411.1 & .8973 & 1. 1873 & 95 \\
\hline 96 & 55.68 & .026644 & 1. 794 & .5573 & 250.80 & 160.6 & 411.4 & .8983 & 1.1873 & 96 \\
\hline 97 & 56.57 & .026677 & 1. 767 & .5658 & 251.36 & 160.3 & 411. 7 & .8993 & 1. 1873 & 97 \\
\hline 98 & 57.47 & .026709 & 1. 741 & .5745 & 251.93 & 160.0 & 412.0 & .9003 & 1. 1873 & 98 \\
\hline 99 & 58.38 & .026742 & 1. 715 & .5832 & 252.49 & 159.8 & 412.3 & .9013 & 1.1873 & 99 \\
\hline 100 & 59.30 & .026775 & 1.689 & .5921 & 253.0 & 159.5 & 412.5 & .9023 & 1. 1872 & 100 \\
\hline 101 & 60.24 & .026808 & 1. 664 & .6010 & 253.6 & 159.2 & 412.8 & .9033 & 1. 1872 & 101 \\
\hline 102 & 61.18 & .026841 & 1.639 & .6101 & 254.2 & 158.9 & 413.1 & .9043 & 1. 1872 & 102 \\
\hline 103 & 62.13 & .026874 & 1. 615 & .6193 & 254.8 & 158.6 & 413.4 & .9053 & 1. 1872 & 103 \\
\hline 104 & 63.10 & 026908 & 1. 591 & .6285 & 255.4 & 158.3 & 413.7 & .9063 & 1. 1872 & 104 \\
\hline
\end{tabular}


TABLE 2.-Properties of saturated 1, 3-butadiene-Continued

\begin{tabular}{|c|c|c|c|c|c|c|c|c|c|c|}
\hline \multirow{2}{*}{$\begin{array}{l}\text { Tempera- } \\
\text { ture, } t\end{array}$} & \multirow{2}{*}{$\begin{array}{l}\text { Abs. } \\
\text { pressure }\end{array}$} & \multicolumn{2}{|c|}{ Specific volume } & \multirow{2}{*}{$\begin{array}{c}\text { Density } \\
\text { vapor } 1 / v_{o}\end{array}$} & \multicolumn{3}{|c|}{ Enthalpy } & \multicolumn{2}{|c|}{ Entropy } & \multirow{2}{*}{$\begin{array}{l}\text { Tempera- } \\
\text { ture, } t\end{array}$} \\
\hline & & $v_{f}$ & $v_{g}$ & & Liquid $h_{f}$ & Evap. $h_{f_{\theta}}$ & Vapor $h_{0}$ & Liquid $s_{f}$ & Vapor $s_{0}$ & \\
\hline${ }^{\circ} \mathrm{F}$ & lb/in..$^{2}$ & $f t^{3} / l b$ & $f t^{3} / l b$ & $l b / f t^{3}$ & $B t u / l b$ & Btullb & $B t u / l b$ & $B t u / l b^{\circ} R$ & $B t u / l b^{\circ} R$ & ${ }^{\circ} \mathrm{F}$ \\
\hline 105 & 64.07 & 0. 026941 & 1. 568 & 0.6379 & 256.0 & 158.0 & 414. 0 & 0.9073 & 1. 1872 & 105 \\
\hline 106 & 65.06 & .026975 & 1. 545 & .6474 & 256.4 & 157.8 & 414. 2 & .9083 & 1. 1872 & 106 \\
\hline 107 & 66. 06 & .027009 & 1. 522 & .6570 & 257.0 & 157.5 & 414.5 & .9093 & 1. 1872 & 107 \\
\hline 108 & 67.07 & .027043 & 1.500 & .6668 & 257.6 & 157.2 & 414.8 & .9103 & 1. 1872 & 109 \\
\hline 109 & 68.09 & .027077 & 1. 478 & .6766 & 258.2 & 156.9 & 415.1 & .9113 & 1. 1872 & 109 \\
\hline 110 & 69.12 & .027112 & 1. 457 & .6865 & 258.8 & 156.6 & 415.4 & .9123 & 1. 1872 & 110 \\
\hline 111 & 70.17 & .027146 & 1. 436 & .6966 & 259.3 & 156.3 & 415.6 & .9133 & 1.1872 & 111 \\
\hline 112 & 71.23 & .027181 & 1. 415 & .7068 & 259.9 & 156.0 & 415.9 & .9143 & 1. 1872 & 112 \\
\hline 113 & 72.29 & .027216 & 1. 395 & .7171 & 260.5 & 155.7 & 416. 2 & .9153 & 1. 1872 & 113 \\
\hline 114 & 73.37 & .027251 & 1. 375 & .7275 & 261.1 & 155.4 & 416.5 & .9163 & 1. 1872 & 114 \\
\hline 115 & 74.47 & .027286 & 1. 355 & .7380 & 261.7 & 155.1 & 416.8 & .9173 & 1. 1872 & 115 \\
\hline 116 & 75.57 & .027322 & 1. 336 & .7487 & 262. 2 & 154.8 & 417. 0 & .9183 & 1. 1872 & 116 \\
\hline 117 & 76.69 & .027358 & 1. 317 & .7595 & 262.8 & 154.5 & 417.3 & .9193 & 1. 1872 & 117 \\
\hline 118 & 77.82 & .027393 & 1. 298 & .7704 & 263.4 & 154.2 & 417.6 & .9203 & 1. 1872 & 118 \\
\hline 119 & 78.96 & .027429 & 1. 280 & .7814 & 264.0 & 153.9 & 417.9 & .9213 & 1. 1873 & 119 \\
\hline 120 & 80.11 & .027466 & 1. 262 & .7926 & 264.6 & 153.6 & 418.2 & .9223 & 1. 1873 & 120 \\
\hline 121 & 81.28 & .027502 & 1. 244 & .8039 & 265.1 & 153.3 & 418.4 & .9233 & 1. 1873 & 121 \\
\hline 122 & 82.46 & .027538 & 1. 227 & .8153 & 265.7 & 153.0 & 418.7 & .9243 & 1. 1873 & 122 \\
\hline 123 & 83.65 & .027575 & 1. 209 & .8268 & 266.3 & 152.7 & 419.0 & .9253 & 1. 1873 & 123 \\
\hline 124 & 84.85 & .027612 & 1. 193 & .8385 & 267.0 & 152.3 & 419.3 & 9263 & 1. 1873 & 124 \\
\hline 125 & 86.07 & .027649 & 1. 176 & .8503 & 267.5 & 152.0 & 419.5 & .9273 & 1. 1873 & 125 \\
\hline 126 & 87.30 & .027687 & 1. 160 & .8623 & 268.1 & 151.7 & 419.8 & .9283 & 1. 1874 & 126 \\
\hline 127 & 88.54 & .027724 & 1. 144 & .8743 & 268.7 & 151.4 & 420.1 & .9293 & 1. 1874 & 127 \\
\hline 128 & 89.80 & .027762 & 1. 128 & .8865 & 269.3 & 151.1 & 420.4 & .9303 & 1. 1874 & 128 \\
\hline 129 & 91.07 & .027800 & 1.112 & .8989 & 269.8 & 150.8 & 420.6 & .9313 & 1. 1874 & 129 \\
\hline 130 & 92.35 & .027838 & 1.097 & .9114 & 270.4 & 150.5 & 420.9 & .9323 & 1. 1874 & 130 \\
\hline 131 & 93.65 & .027877 & 1. 082 & .9240 & 271.1 & 150.1 & 421.2 & .9333 & 1. 1875 & 131 \\
\hline 132 & 94.96 & .027915 & 1. 067 & .9368 & 271.7 & 149.8 & 421.5 & .9343 & 1. 1875 & 132 \\
\hline 133 & 96.28 & .027954 & 1. 053 & .9497 & 272.2 & 149.5 & 421.7 & .9353 & 1. 1875 & 133 \\
\hline 134 & 97.62 & .027993 & 1. 039 & .9628 & 272.8 & 149.2 & 422.0 & .9363 & 1. 1875 & 134 \\
\hline 135 & 98.97 & .028032 & 1. 0246 & .9760 & 273.5 & 148.8 & 422.3 & .9373 & 1. 1875 & 135 \\
\hline 136 & 100.33 & .028072 & 1. 0108 & .9893 & 274.0 & 148.5 & 422.5 & .9383 & 1. 1876 & 136 \\
\hline 137 & 101.71 & .028111 & .9972 & 1. 0028 & 274.6 & 148. 2 & 422.8 & .9392 & 1. 1876 & 137 \\
\hline 138 & 103.10 & .028151 & .9838 & 1.0165 & 275.2 & 147.9 & 423.1 & .9402 & 1. 1876 & 138 \\
\hline 139 & 104.51 & .028191 & .9706 & 1.0303 & 275.9 & 147.5 & 423.4 & .9412 & 1. 1876 & 139 \\
\hline 140 & 105.93 & .028232 & .9576 & 1.044 & 276.4 & 147.2 & 423.6 & .9422 & 1. 1877 & 140 \\
\hline 141 & 107.36 & .028272 & .9449 & 1. 058 & 277.0 & 146.9 & 423.9 & .9432 & 1. 1877 & 141 \\
\hline 142 & 108.81 & .028313 & .9323 & 1. 073 & 277.7 & 146.5 & 424. 2 & .9442 & 1. 1877 & 142 \\
\hline 143 & 110.27 & .028354 & .9199 & 1.087 & 278.2 & 146. 2 & 424.4 & .9452 & 1. 1878 & 143 \\
\hline 144 & 111.75 & .028395 & .9077 & 1.102 & 278.8 & 145.9 & 424.7 & .9462 & 1. 1878 & 144 \\
\hline 145 & 113. 24 & .028437 & .8957 & 1.116 & 279.5 & 145.5 & 425.0 & .9472 & 1. 1878 & 145 \\
\hline 146 & 114. 75 & .028479 & .8839 & 1. 131 & 280.0 & 145.2 & 425.2 & .9482 & 1. 1878 & 146 \\
\hline 147 & 116.27 & .028521 & .8723 & 1.146 & 280.7 & 144.8 & 425.5 & .9492 & 1. 1879 & 147 \\
\hline 148 & 117.81 & .028563 & .8608 & 1. 162 & 281.3 & 144.5 & 425.8 & .9501 & 1. 1879 & 148 \\
\hline 149 & 119.36 & .028606 & .8496 & 1.177 & 281.9 & 144.1 & 426.0 & .9511 & 1. 1879 & 149 \\
\hline 150 & 120.9 & .028648 & .8385 & 1.193 & 282.5 & 143.8 & 426.3 & .9521 & 1. 1880 & 150 \\
\hline 151 & 122.5 & .028691 & .8275 & 1. 208 & 283.1 & 143.5 & 426.6 & .9531 & 1. 1880 & 151 \\
\hline 152 & 124.1 & .028735 & .8167 & 1. 224 & 283.7 & 143.1 & 426.8 & .9541 & 1. 1880 & 152 \\
\hline 153 & 125.7 & .028778 & .8061 & 1. 240 & 284.4 & 142.7 & 427.1 & .9551 & 1. 1881 & 153 \\
\hline 154 & 127.3 & .028822 & .7957 & 1. 257 & 285.0 & 142.4 & 427.4 & .9561 & 1. 1881 & 154 \\
\hline 155 & 129.0 & .028866 & .7854 & 1. 273 & 285.6 & 142.0 & 427.6 & .9571 & 1. 1881 & 155 \\
\hline 156 & 130.6 & .028911 & .7753 & 1. 290 & 286.2 & 141.7 & 427.9 & .9581 & 1. 1882 & 156 \\
\hline 157 & 132.3 & .028956 & .7653 & 1.307 & 286.8 & 141.3 & 428.1 & .9590 & 1. 1882 & 157 \\
\hline 158 & 134.0 & .029001 & .7554 & 1.324 & 287.4 & 141.0 & 428.4 & .9600 & 1. 1883 & 158 \\
\hline 159 & 135.7 & .029046 & .7458 & 1.341 & 288.0 & 140.6 & 428.6 & .9610 & 1. 1883 & 159 \\
\hline
\end{tabular}


TABLE 2.-Properties of saturated 1, 3-butadiene-Continued

\begin{tabular}{|c|c|c|c|c|c|c|c|c|c|c|}
\hline \multirow{2}{*}{$\begin{array}{l}\text { Tempera- } \\
\text { ture, } t\end{array}$} & \multirow{2}{*}{$\begin{array}{c}\text { Abs. } \\
\text { pressure }\end{array}$} & \multicolumn{2}{|c|}{ Specific volume } & \multirow{2}{*}{$\begin{array}{c}\text { Density } \\
\text { vapor } 1 / v_{g}\end{array}$} & \multicolumn{3}{|c|}{ Enthalpy } & \multicolumn{2}{|c|}{ Entropy } & \multirow{2}{*}{$\begin{array}{l}\text { Tempera- } \\
\text { ture, } t\end{array}$} \\
\hline & & $v_{f}$ & $v_{0}$ & & Liquid $h_{f}$ & Evap. $h_{f o}$ & Vapor $h_{。}$ & Liquid $s_{f}$ & Vapor $s_{0}$ & \\
\hline${ }^{\circ} \mathrm{F}$ & $l b / i n .^{2}$ & $f t^{3} / l b$ & $f t^{3} / l b$ & $l b / f t^{3}$ & $B t u / l b$ & $B t u / l b$ & $B t u / l b$ & $B t u / l b^{\circ} R$ & $B t u / l b^{\circ} R$ & ${ }^{\circ} \mathrm{F}$ \\
\hline 160 & 137.4 & 0.029092 & 0.7362 & 1. 358 & 288.6 & 140.3 & 428.9 & 0.9620 & 1. 1883 & 160 \\
\hline 161 & 139.2 & .029138 & .7268 & 1. 376 & 289.3 & 139.9 & 429.2 & .9630 & 1. 1884 & 161 \\
\hline 162 & 140.9 & .029184 & .7175 & 1. 394 & 289.9 & 139.5 & 429.4 & .9640 & 1.1884 & 162 \\
\hline 163 & 142.7 & .029230 & .7084 & 1. 412 & 290.5 & 139.2 & 429.7 & .9650 & 1. 1884 & 163 \\
\hline 164 & 144.5 & .029277 & .6994 & 1. 430 & 291.1 & 138.8 & 429.9 & .9659 & 1. 1885 & 164 \\
\hline 165 & 146.3 & 0.29324 & .6905 & 1. 448 & 291.8 & 138.4 & 430.2 & .9669 & 1. 1885 & 165 \\
\hline 166 & 148.1 & .029372 & .6818 & 1. 467 & 292.3 & 138.1 & 430.4 & .9679 & 1. 1885 & 166 \\
\hline 167 & 149.9 & .029419 & .6731 & 1. 486 & 293.0 & 137.7 & 430.7 & .9689 & 1. 1886 & 167 \\
\hline 168 & 151.8 & .029467 & .6646 & 1.505 & 293.7 & 137.3 & 431.0 & .9699 & 1. 1886 & 168 \\
\hline 169 & 153.7 & .029516 & .6563 & 1. 524 & 294.3 & 136.9 & 431.2 & .9709 & 1. 1887 & 169 \\
\hline 170 & 155.5 & .029565 & .6480 & 1. 543 & 294.9 & 136.5 & 431.4 & .9719 & 1. 1887 & 170 \\
\hline 171 & 157.5 & .029614 & .6399 & 1. 563 & 295.5 & 136. 2 & 431.7 & .9728 & 1. 1887 & 171 \\
\hline 172 & 159.4 & .029663 & .6318 & 1. 583 & 296. 2 & 135.8 & 432.0 & .9738 & 1. 1888 & 172 \\
\hline 173 & 161.3 & .029713 & .6239 & 1. 603 & 296.8 & 135.4 & 432.2 & .9748 & 1. 1888 & 173 \\
\hline 174 & 163.3 & .029763 & .6161 & 1. 623 & 297.4 & 135.0 & 432.4 & .9758 & 1. 1888 & 174 \\
\hline 175 & 165. 2 & .029814 & .6084 & 1. 644 & 298.1 & 134.6 & 432.7 & .9768 & 1. 1889 & 175 \\
\hline 176 & 167. 2 & .029865 & .6008 & 1. 664 & 298.8 & 134.2 & 433.0 & .9778 & 1. 1889 & 176 \\
\hline 177 & 169.2 & .029916 & .5934 & 1. 685 & 299.4 & 133.8 & 433.2 & .9787 & 1.1890 & 177 \\
\hline 178 & 171.3 & .029968 & .5860 & 1. 707 & 300.0 & 133.4 & 433.4 & .9797 & 1. 1890 & 178 \\
\hline 179 & 173.3 & .030020 & .5787 & 1. 728 & 300.6 & 133.1 & 433.7 & .9807 & 1. 1890 & 179 \\
\hline 180 & 175.4 & .030072 & .5715 & 1. 750 & 301.3 & 132.6 & 433.9 & .9817 & 1. 1891 & 180 \\
\hline 181 & 177.4 & .030125 & .5644 & 1. 772 & 301.9 & 132.3 & 434. 2 & .9827 & 1. 1891 & 181 \\
\hline 182 & 179.5 & .030178 & .5575 & 1. 794 & 302.5 & 131.9 & 434.4 & .9837 & 1. 1891 & 182 \\
\hline 183 & 181.7 & .030232 & .5506 & 1.816 & 303. 2 & 131.4 & 434.6 & .9846 & 1. 1892 & 183 \\
\hline 184 & 183.8 & .030286 & .5438 & 1. 839 & 303.9 & 131.0 & 434.9 & .9856 & 1. 1892 & 184 \\
\hline 185 & 185.9 & .030340 & .5371 & 1.862 & 304.5 & 130.6 & 435.1 & .9866 & 1.1892 & 185 \\
\hline 186 & 188.1 & .030395 & .5305 & 1.885 & 305. 2 & 130.2 & 435.4 & .9876 & 1. 1893 & 186 \\
\hline 187 & 190.3 & .030450 & .5239 & 1.909 & 305.8 & 129.8 & 435.6 & .9886 & 1. 1893 & 187 \\
\hline 188 & 192.5 & .030506 & .5175 & 1. 932 & 306.4 & 129.4 & 435.8 & .9896 & 1. 1894 & 188 \\
\hline 189 & 194.7 & 030562 & .5112 & 1. 956 & 307.1 & 129.0 & 436.1 & .9906 & 1. 1894 & 189 \\
\hline 190 & 197.0 & .030619 & .5049 & 1.981 & 307.7 & 128.6 & 436.3 & .9915 & 1. 1894 & 190 \\
\hline 191 & 199. 2 & .030676 & .4987 & 2.005 & 308.3 & 128.2 & 436.5 & .9925 & 1. 1895 & 191 \\
\hline 192 & 201.5 & .030734 & .4926 & 2. 030 & 309.1 & 127.7 & 436.8 & .9935 & 1. 1895 & 192 \\
\hline 193 & 203.8 & .030792 & .4866 & 2. 055 & 309.7 & 127.3 & 437.0 & .9945 & 1. 1895 & 193 \\
\hline 194 & 206.1 & .030850 & .4806 & 2.081 & 310.3 & 126.9 & 437. 2 & .9955 & 1. 1895 & 194 \\
\hline 195 & 208.5 & .030909 & .4748 & 2. 106 & 311.0 & 126.4 & 437.4 & .9964 & 1. 1896 & 195 \\
\hline 196 & 210.9 & 030969 & .4690 & 2. 132 & 311.7 & 126.0 & 437.7 & .9974 & 1. 1896 & 196 \\
\hline 197 & 213.2 & .031029 & .4632 & 2.159 & 312.3 & 125.6 & 437.9 & .9984 & 1. 1896 & 197 \\
\hline 198 & 215.6 & .031090 & .4576 & 2. 185 & 313.0 & 125.1 & 438.1 & .9994 & 1. 1897 & 198 \\
\hline 199 & 218.1 & 031151 & .4520 & 2. 212 & 313.6 & 124.7 & 438.3 & 1. 0004 & 1. 1897 & 199 \\
\hline 200 & 220.5 & .031213 & .4465 & 2. 240 & 315 & 124 & 439 & 1.001 & 1. 190 & 200 \\
\hline 202 & 225.4 & .031337 & .4357 & 2. 295 & 316 & 123 & 439 & 1. 003 & 1. 190 & 202 \\
\hline 204 & 230.5 & .031464 & .4252 & 2. 352 & 317 & 122 & 439 & 1. 005 & 1. 190 & 204 \\
\hline 206 & 235.6 & .031594 & .4149 & 2.410 & 318 & 122 & 440 & 1. 007 & 1. 190 & 206 \\
\hline 208 & 240.8 & .031726 & .4049 & 2.470 & 319 & 121 & 440 & 1. 009 & 1. 190 & 208 \\
\hline 210 & 246.0 & .031861 & .3951 & 2. 531 & 321 & 120 & 441 & 1. 011 & 1. 190 & 210 \\
\hline 212 & 251.4 & .031998 & .3856 & 2. 594 & 322 & 119 & 441 & 1. 013 & 1. 190 & 212 \\
\hline 214 & 256.8 & .032138 & .3763 & 2. 658 & 323 & 118 & 441 & 1. 015 & 1. 190 & 214 \\
\hline 216 & 262.4 & .032281 & .3672 & 2. 732 & 325 & 117 & 442 & 1. 017 & 1. 190 & 216 \\
\hline 218 & 268.0 & .032427 & .3583 & 2. 791 & 326 & 116 & 442 & 1019 & 1. 190 & 218 \\
\hline 220 & 273.7 & .032576 & .3496 & 2.860 & 328 & 115 & 443 & 1. 021 & 1. 190 & 220 \\
\hline 222 & 279.5 & .032728 & .3411 & 2. 931 & 329 & 114 & 443 & 1. 023 & 1. 190 & 222 \\
\hline 224 & 285.4 & .032884 & .3329 & 3.004 & 330 & 113 & 443 & 1. 025 & 1. 190 & 224 \\
\hline 226 & 291.4 & .033044 & .3248 & 3. 079 & 332 & 112 & 444 & 1. 027 & 1. 190 & 226 \\
\hline 228 & 297.5 & .033207 & .3168 & 3156 & 333 & 111 & 444 & 1. 029 & 1. 190 & 228 \\
\hline
\end{tabular}


TABLE 2.-Properties of saturated 1, 3-butadiene-Continued

\begin{tabular}{|c|c|c|c|c|c|c|c|c|c|c|}
\hline \multirow{2}{*}{$\begin{array}{l}\text { Tempera- } \\
\text { ture, } t\end{array}$} & \multirow{2}{*}{$\begin{array}{l}\text { Abs. } \\
\text { pressure }\end{array}$} & \multicolumn{2}{|c|}{ Specific volume } & \multirow{2}{*}{$\begin{array}{l}\text { Density } \\
\text { vapor } 1 / v_{q}\end{array}$} & \multicolumn{3}{|c|}{ Enthalpy } & \multicolumn{2}{|c|}{ Entropy } & \multirow{2}{*}{$\begin{array}{l}\text { Tempera- } \\
\text { ture, } t\end{array}$} \\
\hline & & $v_{f}$ & $v_{0}$ & & Liquid $h_{f}$ & Evap. $h_{f a}$ & Vapor $h_{0}$ & Liquid $s_{f}$ & Vapor $s_{\theta}$ & \\
\hline${ }^{\circ} \mathrm{F}$ & lb/in.2 ${ }^{2}$ & $f t^{3} / l b$ & $f t^{3} / l b$ & $l b / f t^{3}$ & $B t u / l b$ & $B t u / l b$ & $B t u / l b$ & $B t u / l b^{\circ} R$ & $B t u / l b^{\circ} R$ & ${ }^{\circ} \mathrm{F}$ \\
\hline 230 & 303. 7 & 0.03337 & 0.3091 & 3. 235 & 334 & 110 & 444 & 1. 031 & 1. 190 & 230 \\
\hline 232 & 310.0 & .03354 & .3015 & 3. 817 & 336 & 109 & 445 & 1. 033 & 1. 190 & 232 \\
\hline 234 & 316.3 & .03372 & .2941 & 3. 400 & 338 & 107 & 445 & 1. 035 & 1. 190 & 234 \\
\hline 236 & 322.8 & 03390 & .2868 & 3.487 & 339 & 106 & 445 & 1. 037 & 1. 190 & 236 \\
\hline 238 & 329.4 & .03408 & .2797 & 3.575 & 341 & 105 & 446 & 1. 039 & 1. 190 & 238 \\
\hline 240 & 336.1 & .03427 & .2727 & 3. 667 & 342 & 104 & 446 & 1. 041 & 1. 190 & 240 \\
\hline 242 & 342.9 & .03447 & .2659 & 3. 761 & 343 & 103 & 446 & 1. 043 & 1.190 & 242 \\
\hline 244 & 349.8 & .03467 & .2592 & 3.858 & 344 & 102 & 446 & 1.045 & 1. 189 & 244 \\
\hline 246 & 356.8 & .03488 & .2526 & 3.959 & 347 & 100 & 447 & 1.047 & 1. 189 & 246 \\
\hline 248 & 363.9 & .03509 & .2462 & 4. 062 & 348 & 99 & 447 & 1. 049 & 1. 189 & 248 \\
\hline 250 & 371.1 & .03531 & .2398 & 4. 170 & 349 & 98 & 447 & 1. 051 & 1. 189 & 250 \\
\hline 252 & 378.5 & .03554 & .2336 & 4. 281 & 351 & 96 & 447 & 1. 053 & 1. 189 & 252 \\
\hline 254 & 385.9 & .03578 & .2274 & 4. 397 & 352 & 95 & 447 & 1. 055 & 1. 189 & 254 \\
\hline 256 & 393.5 & .03603 & .2214 & 4. 517 & 354 & 94 & 448 & 1.057 & 1. 188 & 256 \\
\hline 258 & 401.2 & .03628 & .2155 & 4. 640 & 356 & 92 & 448 & 1. 059 & 1. 188 & 258 \\
\hline 260 & 409 & .0365 & .2097 & 4. 770 & 357 & 91 & 448 & 1. 062 & 1. 188 & 260 \\
\hline 262 & 417 & .0368 & .2039 & 4. 904 & 359 & 89 & 448 & 1. 064 & 1. 187 & 262 \\
\hline 264 & 425 & .0371 & .1982 & 5.045 & 360 & 88 & 448 & 1. 066 & 1. 187 & 264 \\
\hline 266 & 433 & .0374 & .1926 & 5. 192 & 362 & 86 & 448 & 1. 068 & 1. 187 & 266 \\
\hline 268 & 441 & .0377 & .1870 & 5. 347 & 364 & 84 & 448 & 1. 070 & 1. 186 & 268 \\
\hline 270 & 450 & .0381 & .1815 & 5. 51 & 365 & 83 & 448 & 1. 073 & 1. 186 & 270 \\
\hline 272 & 459 & .0384 & .1761 & 5. 68 & 367 & 81 & 448 & 1. 075 & 1. 185 & 272 \\
\hline 274 & 467 & .0388 & .1707 & 5. 86 & 369 & 79 & 448 & 1. 077 & 1. 185 & 274 \\
\hline 276 & 476 & .0392 & .1653 & 6.05 & 370 & 77 & 447 & 1. 079 & 1. 184 & 276 \\
\hline 278 & 485 & .0396 & 1599 & 6.25 & 372 & 75 & 447 & 1. 082 & 1. 183 & 278 \\
\hline 280 & 494 & .0401 & .155 & 6.47 & 375 & 72 & 447 & 1. 084 & 1. 182 & 280 \\
\hline 282 & 504 & .0406 & .149 & 6. 71 & 376 & 70 & 446 & 1. 087 & 1. 181 & 282 \\
\hline 284 & 513 & .0411 & .144 & 6. 96 & 379 & 67 & 446 & 1.090 & 1. 180 & 284 \\
\hline 286 & 523 & .0417 & .138 & 7. 24 & 380 & 65 & 445 & 1. 092 & 1. 179 & 286 \\
\hline 288 & 533 & .0424 & .132 & 7. 55 & 382 & 62 & 444 & 1.095 & 1. 178 & 288 \\
\hline
\end{tabular}

The values of enthalpy for the liquid have been given to hundredths of a Btu/lb where they were well supported by experimental data. In consequence of the rounding off of the values for the vapor and for evaporation, the latter may differ by one in the last place from the difference between the enthalpy of vapor and of liquid.

The values in the table and chart were calculated by methods described in a previous publication [8] after first converting the four empirical equations given in that publication to engineering units.

In computing the figures in the table from the empirical equations, all calculations were carried two digits beyond the number to be finally retained in the table. The values have been checked by differences.

The number of empirical equations used has been kept to a minimum, and exact thermodynamic relations have been used whenever possible.
In consequence, the values given in the table are thermodynamically consistent.

In consideration of the large size of tables for superheated vapor and of the fact that the data for 1,3-butadiene can be represented on a chart nearly within the accuracy of the observed data, the properties of the superheated vapor are presented only in graphical form.

\section{Description of the Mollier Chart}

Five properties, namely, temperature, pressure, specific volume, enthalpy, and entropy are useful in solving thermodynamic problems. These properties may be presented either in a table or on a chart such as first designed by Mollier [9]. Any two of the properties may be plotted as coordinates, but enthalpy usually is chosen as one coordinate as it is the property for which numerical values are of most interest. Any one of the other 
four properties or a function thereof may be used for the other coordinate, depending upon the nature of the problems to be solved and the individual preference of the user. In the present instance, the use of the logarithm of pressure gives a constant percentage accuracy in reading the pressure and utilizes the rectangular paper efficiently. Usually all five properties are included, but for the sake of clarity, the constant volume lines have been omitted from the present Mollier chart. Values for the volume may be derived from figures 3,4 , and 5 .

The logarithms of pressures from 3 to $300 \mathrm{lb} / \mathrm{in}^{2}$ have been plotted as ordinates. Two scales along the abscissa have been used to represent enthalpies, the one at the left for the liquid, and the one at the right for the vapor. The curves representing the saturated vapor and the saturated liquid are so marked. The area to the left of the curve for saturated liquid represents the subcooled liquid for which no data are available. It should be noted that data for other substances indicate that the constant temperature lines are practically vertical over this area. The area between the curves for saturated liquid and for saturated vapor represents mixtures of vapor and liquid. It has not been charted and the distance between the two saturation lines has no physical significance. The area at the right represents superheated vapor up to $300^{\circ} \mathrm{F}$.

The chart as originally drawn is 80 by $98 \mathrm{~cm}$ (approximately $31 \frac{1}{2} \times 38 \frac{1}{2}$ in.). The mid points of each coordinate line were located by means of a beam compass and an accurate scale, and the coordinate lines drawn in by means of a parallel straight edge. Various checks indicate that the coordinates are drawn with an accuracy of 0.2 or $0.3 \mathrm{~mm}$, corresponding to about 0.05 $\mathrm{Btu} / \mathrm{lb}$ and to about two parts in 1,000 of the pressure.

Over 600 points were plotted so that each curve was drawn through a considerable number of suitably chosen points by means of a spline and weights. Errors in plotting larger than 0.2 or $0.3 \mathrm{~mm}$ became evident due to the difficulty of fitting the spline to the points with a reasonable number of weights. This estimate of the accuracy of plotting is supported by the fact that out of values read from 20 points on the original drawing, the largest discrepancy between the chart and calculated values was $0.05 \mathrm{Btu} / \mathrm{lb}(0.3$ $\mathrm{mm}$ ) and $0.0008 \mathrm{Btu} / \mathrm{lb}{ }^{\circ} \mathbf{R}$ in entropy units $(0.3 \mathrm{~mm})$.

\section{Use of Tables and Charts}

The usefulness of the table and Mollier chart is based largely on three properties of the thermodynamic function enthalpy (sometimes called heat content). The three properties referred to are (1) In any process that occurs at constant pressure the increase in enthalpy is equal to the heat added; (2) in adiabatic compression - that is, at constant entropy-the work done by the compressor is equal to the change in enthalpy of the fluid between intake and discharge; and (3) in a throttling process, such as occurs at an expansion valve, the increase in enthalpy is equal to the heat added. Therefore, when no heat is added the enthalpy is constant.

Two problems that illustrate the application of the three principles mentioned above, through use of the chart, will now be discussed.

Problem 1. Butadiene is admitted into a distillation unit as liquid under an absolute pressure of about $80 \mathrm{lb} / \mathrm{in} .^{2}$ and at a temperature of $70^{\circ} \mathrm{F}$. It is to be converted under the same pressure to gas at $120^{\circ} \mathrm{F}$. How much heat must be added per pound of butadiene?

Due to lack of experimental data, the chart does not give values of enthalpy for the subcooled liquid; but from data on other substances it may be inferred that the change of enthalpy with pressure for subcooled liquid butadiene at constant temperature is so small that for all practical purposes the value at $80 \mathrm{lb} / \mathrm{in}^{2}$ absolute and $70^{\circ}$ $\mathrm{F}$ is the same as the value $236.5 \mathrm{Btu} / \mathrm{lb}$ for saturated liquid at $70^{\circ} \mathrm{F}$. The chart indicates that vapor leaving the still at $80 \mathrm{lb} / \mathrm{in}^{2}{ }^{2}$ absolute pressure and $120^{\circ} \mathrm{F}$ is practically saturated vapor and that its enthalpy is $418.2 \mathrm{Btu} / \mathrm{lb}$. The difference $418.2-236.5=181.7$ Btu per lb must be supplied to the butadiene. The answer should be affected only slightly by the presence of appreciable amounts of other $\mathrm{C}_{4}$ hydrocarbons, as they have properties very similar to butadiene, but the presence of dissimilar compounds renders the answer unreliable in such problems. The heat of solution is involved in evaporation of mixtures that for dissimilar substances may differ appreciably from the heat of evaporation for butadiene.

Although correct answers can be obtained only when these data are applied to the pure monomer 
of 1,3-butadiene, yet in many of the problems to be solved, mixtures of the monomer with polymer or with other compounds are encountered. In some cases if the composition of the mixture is known, a judicious use of the chart may lead to a sufficiently approximate answer.

In the following problem, which involves such a mixture, the results of two methods of calculation with the use of the chart are compared with the results of a third method of calculation in which data are used from tables or charts appropriate for each component of the mixture.

The correct answer to this problem could be obtained only from measurements made on the mixture itself. Such data are rarely available, but the results obtained by the third method are believed to approach the correct answer most closely.

Problem 2. In some processes a gaseous mixture saturated with water vapor and containing about 90 percent of butadiene and 8 percent of ammonia is encountered. The initial temperature and pressure of this mixture will be assumed to be $78^{\circ} \mathrm{F}$ and $25 \mathrm{lb} /$ in. $^{2}$, respectively. In actual practive the pressure of this mixture is increased only a few pounds per square inch through compression. We will assume for the present problem, however, a compression ratio of two, i. e. a discharge pressure of $50 \mathrm{lb} / \mathrm{in}^{2}$, for the sake of sizable differences on the Mollier chart that may be read with sufficient accuracy for the comparison of three methods of calculation. The problem will consist in finding (1) the work performed by the compressor (2) the temperature of the discharged gas and (3) the increase in work of compression caused by pressure drop through valves or other constrictions.

In the first method, the procedure is to calculate the results for pure butadiene as a rough approximation to the results for the mixture.

The initial condition is represented on the Mollier chart by the intersection between the pressure ordinate $25 \mathrm{lb} / \mathrm{in}^{2}$ and the constant temperature $78^{\circ} \mathrm{F}$ interpolated between the dotted curves for $70^{\circ} \mathrm{F}$ and for $80^{\circ} \mathrm{F}$. This intersection corresponds to an enthalpy of 408.0 $\mathrm{Btu} / \mathrm{lb}$ and an entropy of 1.209. The intersection of this value for entropy with the pressure ordinate $50 \mathrm{lb} / \mathrm{in.}^{2}$ is at an enthalpy of $421.5 \mathrm{Btu} / \mathrm{lb}$ and a temperature of $120^{\circ} \mathrm{F}$. Then for pure butadiene (1) the work of compression is $421.5-$
$408.0=13.5 \mathrm{Btu} / \mathrm{lb}$ and (2) the discharge temperature is $120^{\circ} \mathrm{F}$.

A second method, which gives a better estimate of the work of compression, uses the chart by taking account of the fact that for equal compression ratios with the same inlet temperature, the work of compression per mole varies only 10 or 20 percent from substance to substance, whereas the work per unit mass varies over a wide range. On the molar basis the calculation is as follows:

$0.9 \mathrm{lb}$ butadiene $=0.9 / 54=0.01665 \mathrm{lb}$ mole.

$0.08 \mathrm{lb}$ ammonia $=0.08 / 17=0.00471 \mathrm{lb}$ mole.

$0.02 \mathrm{lb}$ water $\quad=0.02 / 18=0.00111 \mathrm{lb}$ mole.

$$
\text { Total__._. . . } 0.02247 \text { lb mole. }
$$

$1 \mathrm{lb}$ butadiene $=1 / 54=0.01852$ mole.

Work of compression for 1 lb of mixture is

$$
13.5 \times 0.02247 / 0.01852=16.4 \mathrm{Btu} / \mathrm{lb} .
$$

The third and probably the best method for estimating the work of compression is to sum up the work for compressing the various components separately through the same compression ratio from their initial partial pressures. Fortunately, such procedure is possible for the mixture presented in this problem, as tables of the properties of ammonia [10] and of steam [11] are both available.

The partial pressures are for butadiene 22.5 $\mathrm{lb} / \mathrm{in}^{2}{ }^{2}$, for ammonia $2.0 \mathrm{lb} / \mathrm{in}^{2}{ }^{2}$, and for water the saturation pressure at $78^{\circ} \mathrm{F}$, which is given by Keenan and Keyes in their table 1 as $0.4747 \mathrm{lb} / \mathrm{in}^{2}$ Using the same procedure as before for butadiene at $25 \mathrm{lb} /$ in. $^{2}$ initial pressure, and the same initial temperature $78^{\circ} \mathrm{F}$ and compression ratio, two, one finds the same values for the work and the discharge temperature, for example, 13.5 Btu/lb and $120^{\circ} \mathrm{F}$, respectively.

The ammonia tables mentioned do not include pressures below $5 \mathrm{lb} /$ in. $^{2}$ but the work of compression for a compression ratio of 2 and initial temperature $78^{\circ} \mathrm{F}$ is nearly independent of initial pressure. For initial pressures of 5 and $10 \mathrm{lb} / \mathrm{in}^{2}$, the work of compression is respectively 46.8 and 46.7 Btu/lb, the discharge temperature in both cases being $170.5^{\circ} \mathrm{F}$. One may assume the work of compression to be $46.8 \mathrm{Btu} / \mathrm{lb}$ for an initial pressure of $2 \mathrm{lb} / \mathrm{in}^{2}$, and the discharge temperature $170^{\circ} \mathrm{F}$.

The steam tables by Keenan and Keyes give 
for the enthalpy and entropy of saturated steam at $78^{\circ} \mathrm{F}$, respectively $1,095.8 \mathrm{Btu} / \mathrm{lb}$ and 2.0416 $\mathrm{Btu} / \mathrm{lb}{ }^{\circ} \mathrm{R}$. The discharge pressure is $2 \times 0.4747=$ $0.95 \mathrm{lb} / \mathrm{in}^{2}$. For convenience we will use the rounded pressure $1 \mathrm{lb} / \mathrm{in}^{2}$. The enthalpy and temperature corresponding to an entropy of 2.0416 and a pressure of $1 \mathrm{lb} / \mathrm{in}^{2}$ are $1144.1 \mathrm{Btu} / \mathrm{lb}$ and $186^{\circ} \mathrm{F}$, respectively. The work is then $1144.1-$ $1095.8=48.3 \mathrm{Btu} / \mathrm{lb}$.

The work of compression for the mixture is then,

$$
\begin{gathered}
(0.9 \times 13.5)+(0.08 \times 46.8)+(0.02 \times 48.3)= \\
16.8 \mathrm{Btu} / \mathrm{lb},
\end{gathered}
$$

and the discharge temperature is,

$$
\begin{gathered}
(0.9 \times(120-78))+(0.08 \times(170-78))+ \\
(0.02 \times(186-78))+78=125^{\circ} \mathrm{F} .
\end{gathered}
$$

We now have three values calculated for the work of compression, namely:

(1) For pure butadiene, 13.5 Btu/lb.

(2) For the mixture, using the chart for butadiene together with the assumption that the work of compression per mole is the same for different substances, $16.4 \mathrm{Btu} / \mathrm{lb}$.

(3) For the mixture using the appropriate chart or table for each component of the mixture, 16.8 Btu/lb.

This last method would be expected to give the nearest to the correct answer, but cannot always be used as data may be lacking on some of the components.

The discharge temperature $\left(120^{\circ} \mathrm{F}\right)$ for pure butadiene is in this case lower than that $\left(125^{\circ} \mathrm{F}\right)$ for the mixture. This is due to the fact that the molar specific heats of ammonia and steam are less than that for butadiene.

The additional work that the compressor must perform in consequence of a pressure drop due to throttling through valves or other constrictions may be estimated by employing the third property previously mentioned for enthalpy, i. e. the constancy of enthalpy during a throttling process when no heat is added or removed. The loss is very nearly proportional to the pressure drop and may be calculated by multiplying the work per (lb/in. ${ }^{2}$ ) by the pressure drop. If on the Mollier chart we follow from the point representing intake conditions $\left(78^{\circ} \mathrm{F}\right.$ and $25 \mathrm{lb} /$ in. $\left.^{2}\right)$ downward along the constant enthalpy abscissa $(408.0 \mathrm{Btu} / \mathrm{lb})$ to $24 \mathrm{lb} / \mathrm{in} .,^{2}$ the new entropy is 1.210 . If this constant entropy line is followed back to $25 \mathrm{lb} / \mathrm{in}^{2}$ an enthalpy of $(409.0 \mathrm{Btu} / \mathrm{lb})$ is obtained. The additional work that the compressor must perform in consequence of a $1 \mathrm{lb} / \mathrm{in}^{2}{ }^{2}$ pressure drop in the suction line is $409-408=1 \mathrm{Btu} / \mathrm{lb}$. By a similar process one finds that $1 \mathrm{lb} / \mathrm{in}^{2}{ }^{2}$ pressure drop on the discharge side where the pressure is $50 \mathrm{lb} / \mathrm{in}^{2}$ causes the compressor to perform extra work amounting to $0.5 \mathrm{Btu} / \mathrm{lb}$. In the case where the total compression amounts to only a few pounds, losses due to pressure drop may become an important portion of the whole.

\section{Graphs of Certain Properties of 1, 3-Butadiene}

A number of smaller graphs of the properties of butadiene are presented in this publication (1) to furnish data not readable directly from the Mollier chart and (2) to give the reader a clearer picture of the basis for, and relationships between these data. Figure 1 in which enthalpy (Btu/lb) is plotted vs temperature $\left({ }^{\circ} \mathrm{F}\right)$ is especially in the latter class.

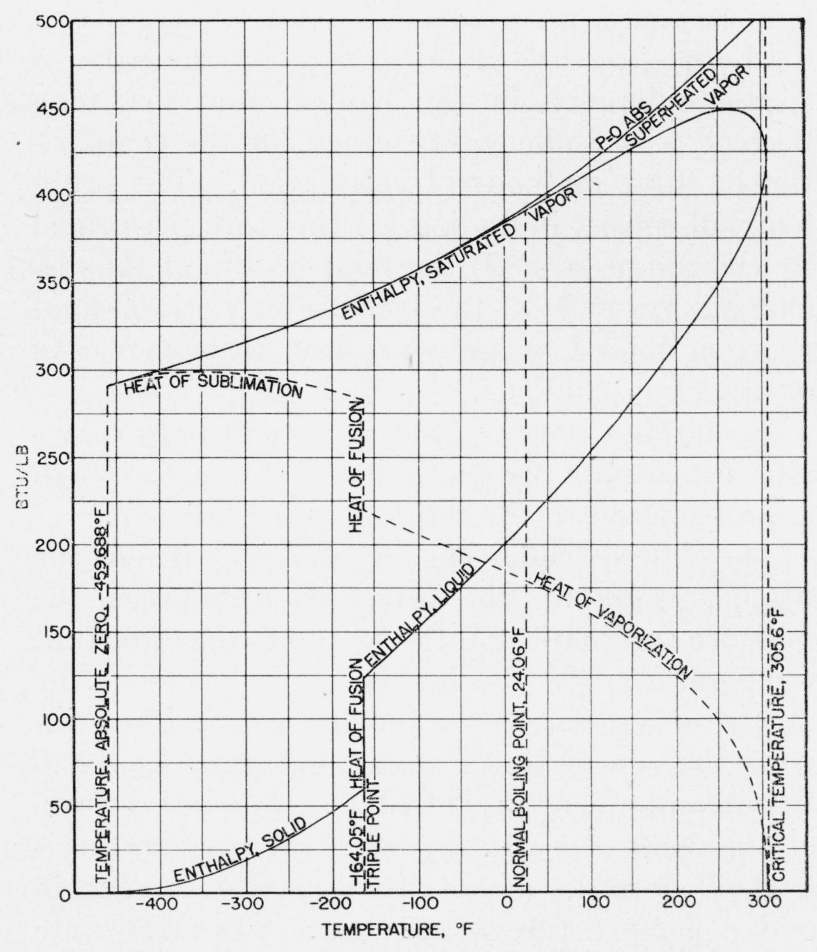

FIGURE 1.-Enthalpy of saturated 1, 3-butadiene. 
As will be noted in this figure, the value for the enthalpy of the crystalline solid has been taken as zero. This has been done for two reasons, namely, (1) except in problems involving chemical reactions no negative numbers are encountered with such choice (2) as many of the theoretical calculations of thermodynamics are based on the theorem that the entropy of crystalline substances is zero at the absolute zero of temperature, the zero for entropy was chosen for this state, and it seemed appropriate to assume the same state for the zero of enthalpy.

This choice of zero state is satisfactory for all the problems for which this publication was designed. A value for the heat of formation from the elements [12] may be added to the enthalpies used here, but its assignment was considered beyond the scope of this publication. As illustrated by the continuous curve in the lower left hand corner of figure 1, the enthalpy for solid butadiene gradually increases with temperature up to the triple point $\left(-164.05^{\circ} \mathrm{F}\right)$ then increases through fusion to liquid without any change in temperature. When the liquid is warmed, the enthalpy again changes with temperature at a rate that gradually increases. The normal boiling point is reached at $24.06^{\circ} \mathrm{F}$, in other words the vapor pressure has increased to one standard atmosphere. With further warming of the liquid, the enthalpy continued to increase at a rate that becomes infinite at the critical temperature $305.6^{\circ} \mathrm{F}$, although the enthalpy itself is finite (about $421 \mathrm{Btu} / \mathrm{lb}$ ).

The heat of vaporization is zero at the critical temperature, but increases to finite values as the temperature is decreased (see dotted curve fig. 1). The rate of increase at the critical is infinite but finite at lower temperatures. At the triple point the heat of sublimation exceeds the heat of vaporization by an amount equal to the heat of fusion. At lower temperatures the heat of sublimation increases to a maximum and then decreases. As near as can be deduced from values of $C_{p}{ }^{\circ}$ from this formulation [8] together with the assumption that $C_{p}{ }^{\circ}$ for the vapor approaches asymptotically toward $4 R$ at absolute zero, the enthalpy of the vapor and consequently the heat of sublimation at absolute zero is about $287 \mathrm{Btu} / \mathrm{lb}$. The enthalpy of the saturated vapor is the sum of the enthalpy for the solid and heat of sublimation, or at higher temperatures the sum of the enthalpy for

Thermodynamic Properties of 1, 3-Butadiene the liquid and the heat of vaporization. The enthalpy of saturated vapor increases with temperature without any break at the triple point, passes through a maximum at about $265^{\circ} \mathrm{F}$ and joins up with the enthalpy of the liquid at the critical temperature. The enthalpy of the vapor at zero pressure, which is the same as that at the standard state (ideal gas at 1 atmos), is not distinguishable from the enthalpy of the saturated vapor at low temperatures, but at higher temperatures the difference calculated from the equation of state increases so that the enthalpy at $p=0$ increases steadily with temperature without any peculiar turn at the critical temperature as exhibited for the saturated vapor.

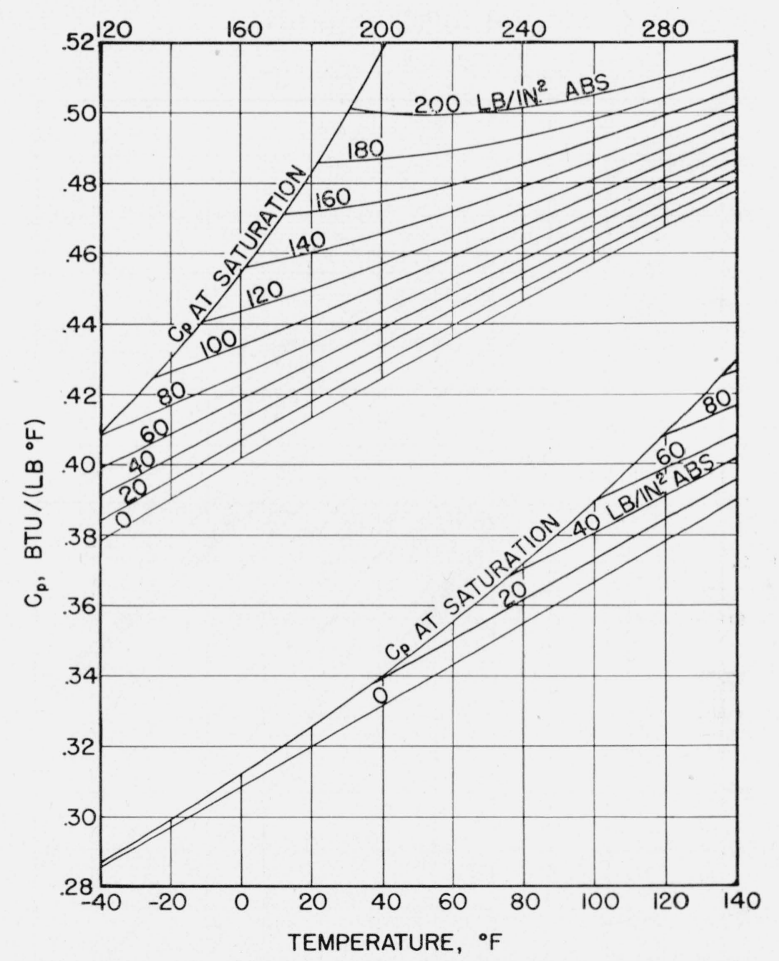

FIGURE 2.-Specific heat at constant pressure of gaseous 1, 3-butadiene.

Figure 2 is a plot of $C_{p}$ the specific heat of the vapor at various pressures from 0 absolute to $200 \mathrm{lb} /$ in. $^{2}$ abs ás a function of temperature expressed in degrees $\mathrm{F}$. The values of $C_{p}$ at zero pressure are calculable from spectroscopic data but were actually calculated from an empirical equation [8] and the values at other pressures can be deduced from these by using the equation of state. The temperatures at the bottom of the graph apply to the lower set of curves, those at the 
top to the upper set, with the two sets overlapping in the range $120^{\circ}$ to $140^{\circ} \mathrm{F}$. The curve for 200 $\mathrm{lb} /$ in. $^{2}$ shows a minimum in $C_{p}$ for the vapor, and if the curves were computed for higher pressures, these minimums would be more pronounced.

Figure 3 shows values of the quantity $P v / R T$ (which is unity for an ideal gas) as a function of pressure for saturated vapor and for superheated vapor at various temperatures. It may be noted that at the highest pressure plotted, $120 \mathrm{lbs} / \mathrm{in}^{2}$ the value of $P v$ for saturated vapor is more than 16 percent less than the value for an ideal gas, but that at low pressure, the values of $P v$ approach $R T$, the approach being closer for the higher temperatures.

Figure 4 is a plot of the quantity $\frac{1-P v / R T}{P\left(l b s / i n^{2}\right)} 10^{4}$

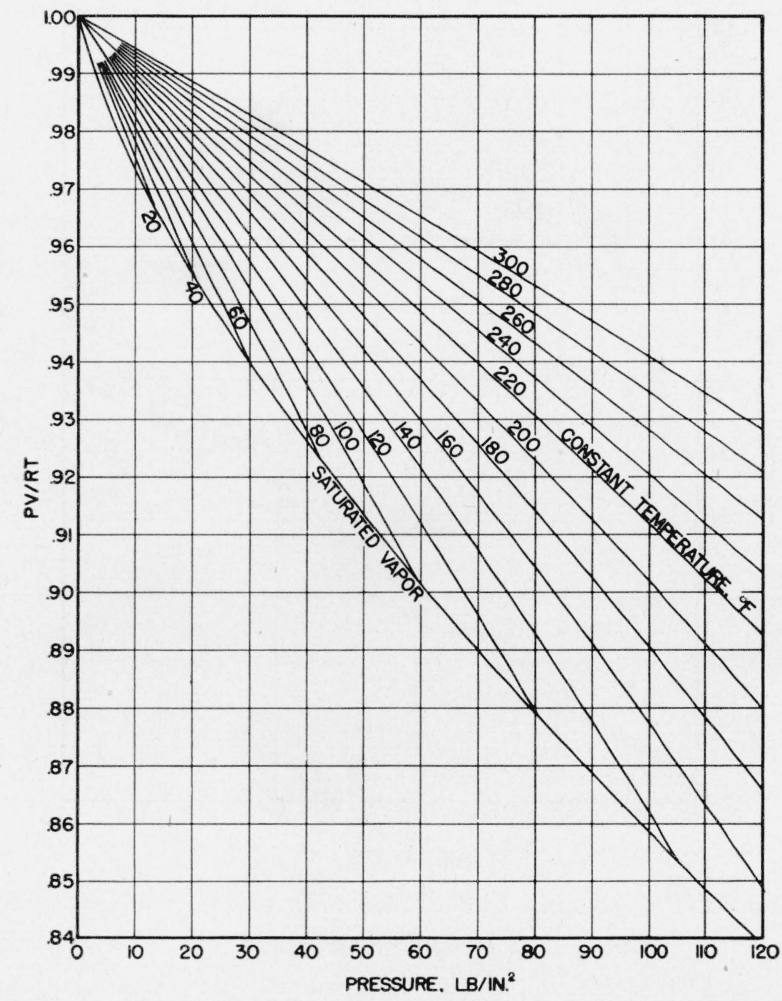

FIgURE 3.-Pressure-tolume-temperature relations for gaseous 1, 3-butadiene.

(which is equal to zero for an ideal gas) for the vapor, as a function of the absolute pressure for various temperatures from $-40^{\circ}$ to $180^{\circ} \mathrm{F}$. The quantity plotted never reaches the value for an ideal gas, but approaches it as the pressure is decreased or as the temperature is raised.

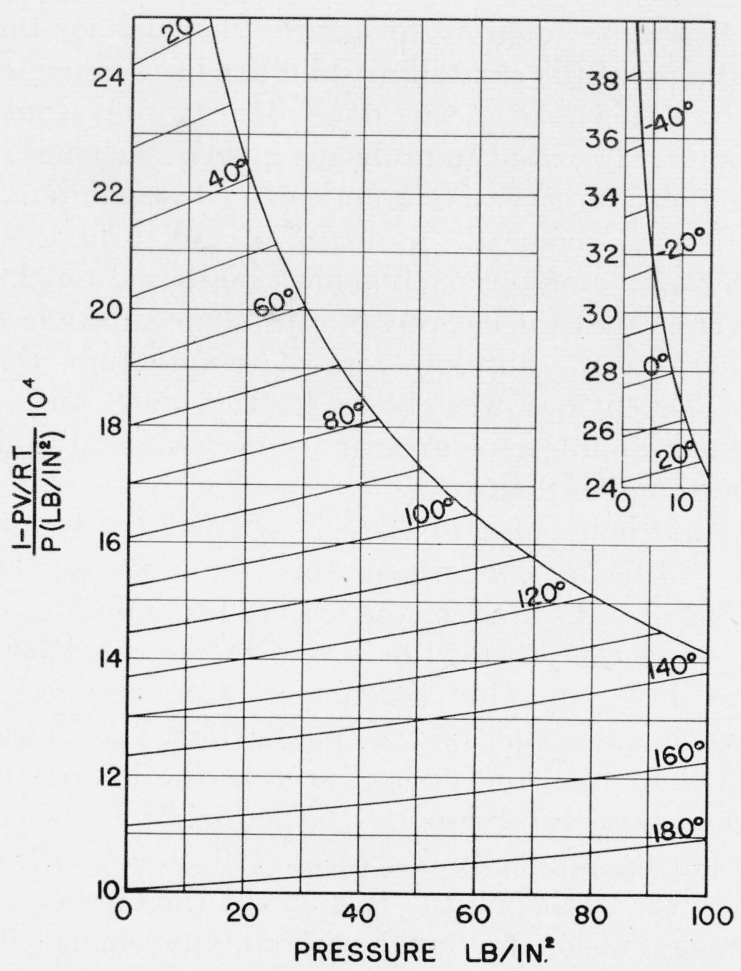

Figure 4.-Pressure-volume-temperature relations for gaseous 1, 3-butadiene.

For use at higher pressures and temperatures the coordinates used in figure 4 require too large a page for accurate presentation of the data. This difficulty is overcome in figure 5 by plotting the product $\frac{1-P v / R T}{P\left(\mathrm{lb} / \mathrm{in} .^{2}\right)}\left(\frac{T}{100}\right)^{3}$ as ordinate vs pressure as abscissa. Through the use of these coordinates accurate values of $P v / R T$ may be obtained over the whole pressure and temperature range although more calculation is involved than in using figure 4. The isotherms cross each other as well as the saturation curve at low pressures and temperatures. In this region the isotherms have been omitted and the use of figure 4 will be less ambiguous.

An acknowledgment with thanks is given to Martin T. Wechsler for calculating the values appearing in the table and to Robert E. McCoskey for calculating the data needed for the Mollier chart and for drawing this chart. 


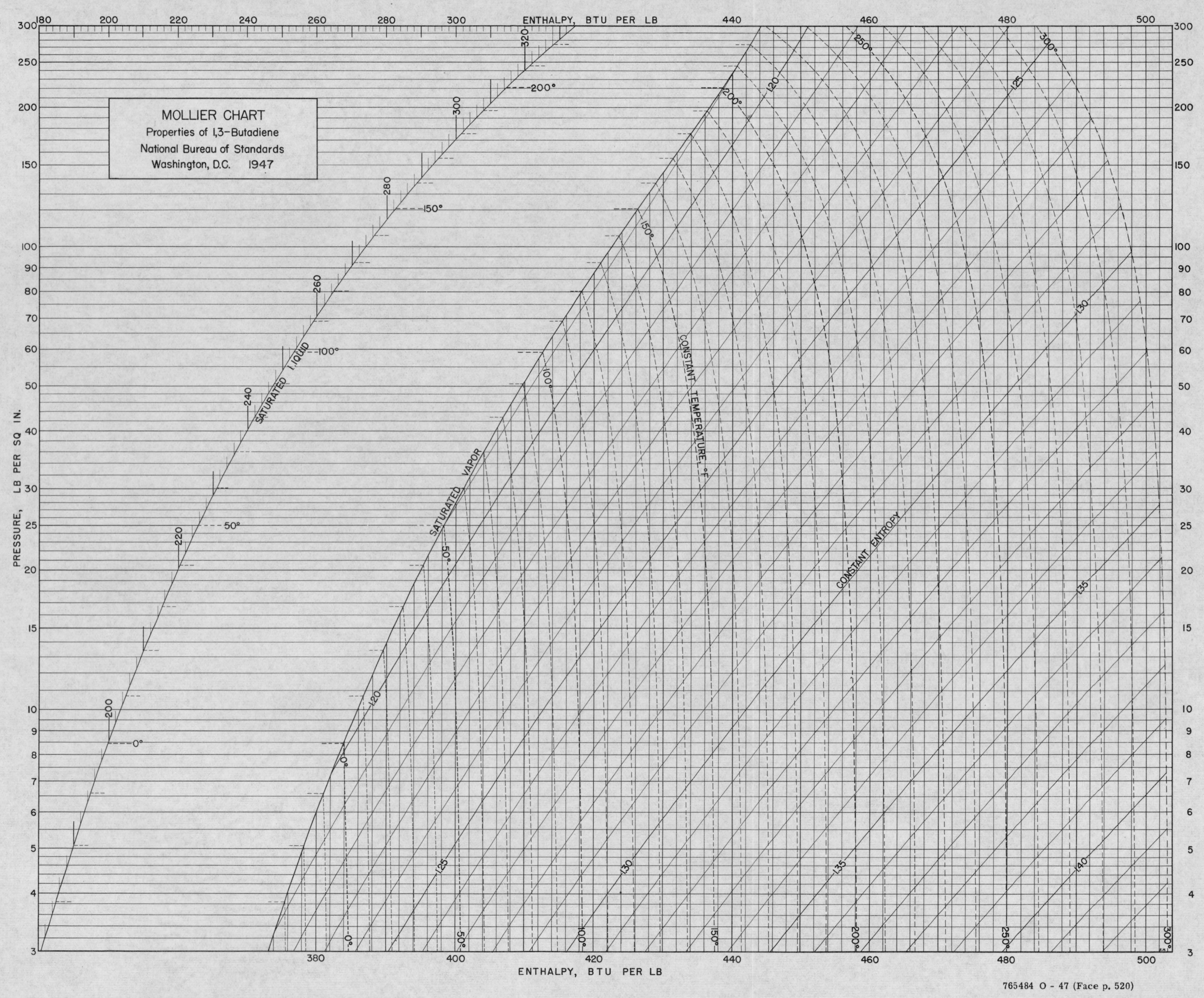




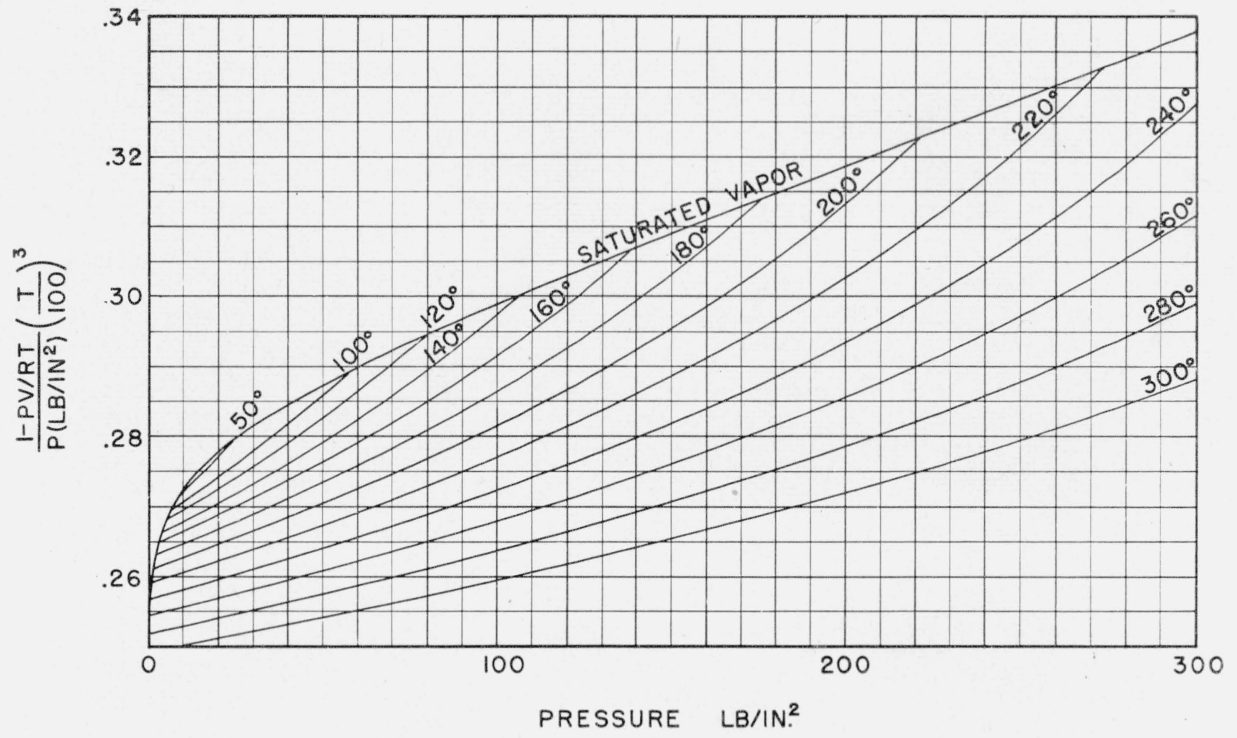

Figure 5.-Pressure-volume-temperature relations for gaseous 1. 3-butadiene.

\section{References}

[1] NBS Letter Circular LC710 (1942).

[2] NBS Letter Circular LC736 (1943).

[3] George K. Burgess, BS J. Research 1, 635 (1928) RP22.

[4] Am. Petroleum Inst. Research Project 44, Selected values of properties of hydrocarbons (Revised, March 31, 1945).

[5] The First International Conference on Steam Tables, Mech. Eng. 52, 120 (1930).

[6] Travaux at Memories Bureau International Poids et Mesures 12, Third Conference Int. Committee, p. 66 (1902).
[7] Russell B. Scott and Jane W. Mellors, J. Research NBS 34, 243 (1945) RP1640.

[8] Russell B. Scott, Cyril H. Meyers, Robert D. Rands, Jr., Ferdinand G. Brickwedde, and Norman Bekkedahl, J. Research NBS 35, 39 (1945) RP1661.

[9] R. Mollier, Zts. d Ver. Deutscher Ing. 48, 271 (1904).

[10] NBS Circular C142 (1923).

[11] J. H. Keenan and F. G. Keyes, Thermodynamic properties of steam (John Wiley and Sons, New York, N. Y., 1936).

[12] J. G. Aston, George Szasz, H. W. Woolley, and F. G. Brickwedde, J. Chem. Phys. 14, 67 (1946).

Washington, July 28, 1947. 